Imperial/TP/92-93/39

\title{
Quantum Logic and the Histories Approach to Quantum Theory
}

\author{
C.J. Isham \\ Blackett Laboratory \\ Imperial College \\ South Kensington \\ London SW7 2BZ \\ United Kingdom
}

August 1993

\begin{abstract}
An extended analysis is made of the Gell-Mann and Hartle axioms for a generalised 'histories' approach to quantum theory. Emphasis is placed on finding equivalents of the lattice structure that is employed in standard quantum logic. Particular attention is given to 'quasi-temporal' theories in which the notion of time-evolution is less rigid than in conventional Hamiltonian physics; theories of this type are expected to arise naturally in the context of quantum gravity and quantum field theory in a curved space-time. The quasi-temporal structure is coded in a partial semi-group of 'temporal supports' that underpins the lattice of history propositions. Non-trivial examples include quantum field theory on a non globally-hyperbolic spacetime, and a simple cobordism approach to a theory of quantum topology.

It is shown how the set of history propositions in standard quantum theory can be realised in such a way that each history proposition is represented by a genuine projection operator. This provides valuable insight into the possible lattice structure in general history theories, and also provides a number of potential models for theories of this type.
\end{abstract}




\section{Introduction}

\subsection{Preliminary remarks}

A good illustration of the great difficulty encountered when attempting to construct a fully comprehensive account of quantum gravity is the striking lack of anything remotely resembling an 'axiomatic' scheme for such a theory. The point at stake is not one of mathematical rigour per se, but rather the need for a clear statement about what one is really trying to do. This uncertainty is reflected in the wide range of declared views on how to approach the subject and, indeed, on what would constitute a successful resolution of the problem.

The lack of any quasi-axiomatic scheme is regretable, but hardly surprising, not least because of the frequently expressed view that the well-developed space-time concepts of classical general relativity may be inappropriate at scales of the Planck length and time. One example is the well-known 'problem of time' that arises in attempts to construct a canonical theory of quantum gravity t. This problem first appears in the canonical approach to classical general relativity where the notion of time as an external parameter disappears and has to be recovered in some way from the internal contents of the system. In the analogous quantum theory opinions differ on whether (i) the concept of 'time' is fundamental; or (ii) our normal idea of time is a coarse-grained concept that emerges only above the Planck scale in a theory of quantum gravity that is fundamentally 'timeless'. In the former case, a major issue is whether the identification of an internal time should be made before, or after, quantisation. Not unsurprisingly, the problem of time is of major importance in quantum cosmology, particularly in attempts to describe the origin of the universe as some type of quantum-mechanical event.

One of the more adventurous suggestions for resolving this issue was made recently by Gell-Mann and Hartle in the context of the 'consistent histories' approach to quantum theory. The consistent histories programme for standard quantum theory is currently generating substantial interest, inspired mainly by a major series of papers by Gell-Mann and Hartle 17, 8, 6, 14, 15, 9, 16] which partly follow on from, and are partly independent of, seminal work by Griffiths [11] and by Omnès [30, 31, 32, 33, 34, 35].

This new perspective on quantum theory is of importance for a variety of reasons, not least of which is the possibility it affords of escaping the thralls of the Copenhagen interpretation with its infamous measurement problem and associated concept of statevector reduction induced by an external observer. Whether or not this hope is justified has been the subject of much, sometimes acrimonious, debate, although most workers in the field agree that, at the very least, the idea of consistent histories provides valuable insight into the relation between classical physics and quantum physics and, in particular, the sense in which the former may be said to 'emerge' from the latter.

However, the motivation of the present paper is not a desire to elude the clutches of

\footnotetext{
${ }^{1}$ For recent reviews see 20,18 .
} 
Copenhagen epistemology but rather the suggestion of Gell-Mann and Hartle to use the methodology of consistent histories to tackle the problem of time in quantum gravity. At a first glance, it seems odd to address the problem of time using the notion of 'history', a concept that is surely intrinsically temporal? But Gell-Mann and Hartle refute this by asserting that a 'history' need not necessarily be regarded as a time-ordered string of events or propositions: it could appear as a fundamental theoretical entity in its own right.

A simple example [16] of this idea is given by a globally-hyperbolic Lorentzian metric $\gamma$ on a manifold $\mathcal{M}$. Any such space-time can be expressed (in a variety of ways) as a one-parameter $t$ (time) family of Riemannian geometries $g_{t}$ on a spatial manifold $\Sigma$ with, topologically, $\mathcal{M} \simeq \Sigma \times \mathbb{R}$. In this sense, $\gamma$ is a possible history of the universe: i.e., a path $t \mapsto g_{t}$ in the infinite-dimensional space of 3-geometries on $\Sigma$. But suppose now that $\gamma$ is not globally hyperbolic. The notion of time is still well-defined locally on $(\mathcal{M}, \gamma)$ but, since there are no global time functions, it is no longer possible to interpret $\gamma$ as a temporal sequence of three-geometries; however, $\gamma$ may still be perfectly acceptable as a space-time geometry, and hence as a 'generalised history' of the universe. Note that a metric of this type may still possess some spacelike hypersurfaces that divide $\mathcal{M}$ into two pieces and, to that extent, some global time structure remains. This is an example of what I shall call a quasi-temporal situation.

This example of Hartle is a mild one, but it illustrates the main point well: in classical or quantum gravity a 'history' is simply a potential configuration for the universe as a whole, including any quasi space-time structure it may possess. This raises a number of fascinating possibilities in which the class of possible universes is deemed to be something considerably more exotic than a collection of non globally-hyperbolic Lorentzian geometries. An example would be a quantum topology theory in which the histories include different point-set topologies with the idea that the smooth manifold structure of classical general relativity emerges only in some coarse-grained sense.

\subsection{The main problems}

The main challenge, and the focus of the present paper, is to translate these rather vague ideas into a concrete mathematical form. What we seek is a quantum history framework that is as mathematically well-formulated as are, for example, the various well-known axiomatisations of standard quantum theory. Of course, any such formalism must reproduce the standard theory where this is appropriate but, hopefully, the axioms will also admit realisations that are very different. This applies in particular to theories of quantum space-time structure where the mathematical apparatus of standard quantum theory (Hilbert spaces, self-adjoint operators, etc) could well be among the things that 'emerge' at a suitably coarse-grained level.

The approach adopted in the present paper is motivated by the presentation by GellMann and Hartle of a set of generalised axioms for a quantum theory based on histories and decoherence functionals. My aim is to construct a refinement of these axioms using 
techniques drawn from the 'quantum logic' approach to standard quantum theory. Thus we are looking for a history-based, quantum gravity analogue of something like, for example, the Mackey [22] or Jauch-Piron axioms [19, 36]. Following the seminal work of Von Neumann and Birkhoff [41], most approaches to quantum logic 2 have concentrated on the lattice 1 structure of the set $\mathcal{L}$ of propositions concerning the state of a physical system at a single time. In realisations based on a Hilbert space $\mathcal{H}$, the lattice $\mathcal{L}$ is identified with the set $P(\mathcal{H})$ of projection operators on $\mathcal{H}$ (or, equivalently, with the set of all closed linear subspaces of $\mathcal{H}$ ). Time evolution appears as an automorphism of the lattice; in a Hilbert space model this is implemented by the familiar family $t \mapsto U(t):=$ $e^{-i H t}$ of unitary operators. In classical physics the lattice of propositions is a Boolean algebra, but in quantum theory this is no longer the case since distributivity is lost: a feature that seems to accurately reflect most of the peculiar features of the quantum world.

The second ingredient in standard quantum logic is the space $\mathcal{R}$ of quantum states. In a Hilbert space realisation this is identified with the set of density matrices. The different axiomatisations vary on whether the primary focus is placed on $\mathcal{L}$ or on $\mathcal{R}$ but, in all cases, a dual relation is posited between these spaces where the pairing $\langle\rho, P\rangle$ of a state $\rho \in \mathcal{R}$ with a proposition $P \in \mathcal{L}$ is identified physically as the probability that $P$ will be true if the state is $\rho$.

The proposal to use quantum logic in the consistent histories formalism is not new; indeed, Omnès invoked such ideas in his seminal work. However, his main aim was to show that any situation in which histories decohere admits a sensible physical description using standard Boolean logic, whereas my goal is quite different. I am not concerned with the mechanism of decoherence as such-nor with the interpretational nuances of quantum physics - but wish rather to explore the basic mathematical structure that might be assigned to the spaces of histories and decoherence functionals. Thus, in what follows, references to quantum 'logic' should not be read as inferring that physical events must necessarily be described using a non-standard form of logic; the implication is only that the set of histories may possess a type of algebraic structure that is similar to the lattices used in standard quantum logic. In particular, I do not wish to become embroiled in the peristalithic debate about whether logic itself is empirical or a priori!

Most studies of quantum logic have involved propositions at a single time and therefore are not directly applicable to the case in hand. A notable exception is the work of Mittelstaedt and Stachow [24, 25, 39, 40] who developed a general theory of the logic of sequential propositions using sequential conjunctions of the type ' $A$ is true and then $B$ is true and then ...', plus other temporal connectives such as 'or then' and 'sequential implication'. One conclusion was that the appropriate mathematical tool for describing sequential propositions is a Baer- ${ }^{*}$ semiring [39]: a mathematical structure that is closely connected with what is done in standard quantum theory using the history formalism.

\footnotetext{
${ }^{2}$ An excellent general introduction is [2].

${ }^{3}$ One can also employ the weaker concept of an orthomodular partially ordered set; for a recent general exposition see [37]. It should be possible to develop a history analogue of this using the ideas in the present paper.
} 
The approach adopted in the present paper can be considered in some respects as an extension of the work of Mittelstaedt and Stachow in a way that takes more account of the temporal labels and which thereby admits an extension to quasi-temporal situations. A key ingredient is the observation that the statement that a certain universe (i.e., history) is 'realised' is itself a proposition, and therefore the set of all such histories might possess a lattice structure analogous to the lattice of single-time propositions in standard quantum logic. In particular, a history proposition might be representable by a projection operator in some Hilbert space.

It must be emphasised that representing histories with projection operators is precisely what does not happen in the usual approach to standard quantum theory. The central object of interest is the so-called decoherence functional (see (2.8)), in whose calculation the history $\alpha$ corresponding to single-time propositions $\alpha_{t_{1}}, \alpha_{t_{2}}, \ldots, \alpha_{t_{n}}$ at times $t_{1}<t_{2}<\ldots<t_{n}$ is associated with the ' $C$-representation' defined as $\widehat{C}_{\alpha}:=$ $\widehat{\alpha}_{t_{n}}\left(t_{n}\right) \widehat{\alpha}_{t_{n-1}}\left(t_{n-1}\right) \ldots \widehat{\alpha}_{t_{1}}\left(t_{1}\right)$ : a product of (Heisenberg picture) projection operators $\widehat{\alpha}_{t_{k}}\left(t_{k}\right)$ that is usually not itself a projection operator. This confuses the logical analysis of the system. For example, if $\alpha$ and $\beta$ are disjoint thistories the $C$-representative $\widehat{C}_{\alpha \vee \beta}$ of 'history $\alpha$ or history $\beta$ ' is always taken to be $\widehat{C}_{\alpha}+\widehat{C}_{\beta}$ : a claim that is usually justified by an appeal to a path-integral formalism. However, a deeper analysis might want to deal with the problem in a more realisation-independent way, in which case the statement $\widehat{C}_{\alpha \vee \beta}=\widehat{C}_{\alpha}+\widehat{C}_{\beta}$ is reminiscent of the standard quantum-logic result that if $P$ and $Q$ are single-time, disjoint propositions, the projection operator that represents ' $P$ or $Q^{\prime}$ is $\widehat{P}+\widehat{Q}$. But the assignment of $\widehat{C}_{\alpha}+\widehat{C}_{\beta}$ cannot be justified in this simple way if $\widehat{C}_{\alpha}$ or $\widehat{C}_{\beta}$ are not projection operators.

This problem becomes more acute if one asks for the $C$-representation of ' $\alpha$ or $\beta$ ' when $\alpha$ and $\beta$ are not disjoint. The analogous question in quantum logic has a direct answer - the proposition $P \vee Q$ is represented by the operator that projects onto the closure of the linear span of the subspaces onto which $\widehat{P}$ and $\widehat{Q}$ project- -but the answer for histories is not obvious. A similar query attends the common identification of $\widehat{1}-\widehat{C}_{\alpha}$ as the $C$-representative of the proposition that the history is ' $n o t$ ' $\alpha$ '

The challenge in construcing a full axiomatic scheme for a quantum history structure is four-fold:

1. Find a general set of axioms for a quantum history theory that has the same degree of precision as one of the well-known axiomatic schemes for standard Hamiltonian quantum theory. This scheme should be broad enough to admit quantum gravity realisations in which the classical notions of space and time are not applicable.

2. If, as expected, the set of all history propositions has some sort of lattice structure, study the way in which this differs from the standard quantum-logic lattices of fixed-time propositions. In particular, what role is played by any 'quasi-temporal' properties of the system?

\footnotetext{
${ }^{4}$ This notion will be defined properly in Section 2
} 
3. Study the extent to which history propositions can be represented as projection operators on some Hilbert space.

4. Study general ways of constructing realisations of the history axioms.

One of the tasks of the present paper is to construct a history version of standard quantum theory in which every history is represented by a projection operator 5 . The usual logical operations on projection operators can then be applied to place a lattice structure on the set of all history propositions. This provides some justification for asserting that, in any history theory, the set of propositions about the histories should have the structure of a non-distributive lattice, of which good models may be the lattices of projection operators in a Hilbert space.

Of course, the challenge of quantum gravity may require realisations of the general history axioms exist that do not involve Hilbert spaces in any way at all. On the other hand, powerful reconstruction theorems in standard quantum theory show that if the lattice $\mathcal{L}$ of single-time propositions satisfies certain plausible conditions it must necessarily be realised as the lattice of subspaces of some (not necessarily complex) Hilbert space. To avoid the analogue of this result in the history case, the algebraic properties of the set of history propositions and the associated space of decoherence functionals would have to differ in some significant way from those of the standard lattice of single-time propositions and associated space of states.

The plan of the paper is as follows. We start with a short exposition of the way histories are used in standard Hamiltonian quantum theory, and of the axioms proposed by Gell-Mann and Hartle. Then the search for a general set of history axioms begins with the introduction of the notion of a temporal support for use in a theory that may possess only quasi-temporal properties. This is based on the observation that one of the most prominent features of a certain type of history (a history filter: a sort of sequential conjunction) in standard quantum theory is that it can be decomposed into sub-histories in which one sub-history 'follows' another. The claim is that the associated structure of a partial semi-group is still present in the general case where it encodes whatever quasi-temporal properties may be possessed by the system.

It is shown that the space of history filters possesses a natural lattice structure, but this is not the correct one physically and only the $\wedge$ semi-lattice sub-structure can be retained. It is necessary therefore to embed the space of history filters into a larger space of general 'history propositions' that carries the correct lattice structure. To validate this crucial point, section 3 is devoted to the construction of a realisation of the logical structure of standard quantum theory in which all history propositions are represented by projection operators on a new Hilbert space. These results are then used to motivate a tentative set of axioms for a general history theory with temporal supports. Relativistic quantum field theory is discussed briefly in section 4 , mainly as a way of illustrating the

\footnotetext{
${ }^{5}$ To avoid confusion it must be emphasised that the Hilbert space on which the histories are represented by projection operators is not the normal Hilbert space of the quantum theory.
} 
use of temporal supports in a quasi-temporal theory. The paper concludes with a short account of some of the outstanding problems for further research.

\section{The Search for General History Axioms}

\subsection{Histories in standard quantum theory}

It will be helpful to begin by summarising very briefly how 'histories' are understood in the conventional interpretation of an open, Hamiltonian quantum system that is subject to measurements by an external, and essentially classical, observer.

To this end, let $U\left(t_{1}, t_{0}\right)$ denote the unitary time-evolution operator from time $t_{0}$ to $t_{1}$; i.e., $U\left(t_{1}, t_{0}\right)=e^{-i\left(t_{1}-t_{0}\right) H / \hbar}$. Then, in the Schrödinger picture, the density operator state $\rho\left(t_{0}\right)$ at time $t_{0}$ evolves in time $t_{1}-t_{0}$ to $\rho\left(t_{1}\right)$, where

$$
\rho\left(t_{1}\right)=U\left(t_{1}, t_{0}\right) \rho\left(t_{0}\right) U\left(t_{1}, t_{0}\right)^{\dagger}=U\left(t_{1}, t_{0}\right) \rho\left(t_{0}\right) U\left(t_{1}, t_{0}\right)^{-1} \text {. }
$$

Suppose that a measurement is made at time $t_{1}$ of a property represented by a projection operator $P$. Then the probability that the property will be found is

$$
\operatorname{Prob}\left(P=1 ; \rho\left(t_{1}\right)\right)=\operatorname{tr}\left(P \rho\left(t_{1}\right)\right)=\operatorname{tr}\left(P U\left(t_{1}, t_{0}\right) \rho\left(t_{0}\right) U\left(t_{1}, t_{0}\right)^{\dagger}\right)=\operatorname{tr}\left(P\left(t_{1}\right) \rho\left(t_{0}\right)\right)
$$

where

$$
P\left(t_{1}\right):=U\left(t_{1}, t_{0}\right)^{\dagger} P\left(t_{0}\right) U\left(t_{1}, t_{0}\right)
$$

is the Heisenberg picture operator defined with respect to the fiducial time $t_{0}$. If the result of this measurement is kept then, according to the Von Neumann-Lüders 'reduction' postulate, the appropriate density matrix to use for any further calculations is

$$
\rho_{\text {red }}\left(t_{1}\right):=\frac{P\left(t_{1}\right) \rho\left(t_{0}\right) P\left(t_{1}\right)}{\operatorname{tr}\left(P\left(t_{1}\right) \rho\left(t_{0}\right)\right)} .
$$

Now suppose a measurement is performed of a second observable $Q$ at time $t_{2}>t_{1}$. Then, according to the above, the conditional probability of getting $Q=1$ at time $t_{2}$ given that $P=1$ was found at time $t_{1}$ (and that the original state was $\rho\left(t_{0}\right)$ ) is

$$
\operatorname{Prob}\left(Q=1 \mid P=1 \text { at } t_{1} ; \rho\left(t_{0}\right)\right)=\operatorname{tr}\left(Q\left(t_{2}\right) \rho_{\text {red }}\left(t_{1}\right)\right)=\frac{\operatorname{tr}\left(Q\left(t_{2}\right) P\left(t_{1}\right) \rho\left(t_{0}\right) P\left(t_{1}\right)\right)}{\operatorname{tr}\left(P\left(t_{1}\right) \rho\left(t_{0}\right)\right)} .
$$

The probability of getting $P=1$ at $t_{1}$ and $Q=1$ at $t_{2}$ is this conditional probability multiplied by $\operatorname{Prob}\left(P=1 ; \rho\left(t_{1}\right)\right)$, i.e.,

$$
\operatorname{Prob}\left(P=1 \text { at } t_{1} \text { and } Q=1 \text { at } t_{2} ; \rho\left(t_{0}\right)\right)=\operatorname{tr}\left(Q\left(t_{2}\right) P\left(t_{1}\right) \rho\left(t_{0}\right) P\left(t_{1}\right)\right) \text {. }
$$

\footnotetext{
${ }^{6}$ For typographical ease, 'hats' will not be placed on operators until it becomes necessary to distinguish propositions from the projection operators that represent them.
} 
Generalising to a sequence of measurements of propositions $\alpha_{t_{1}}, \alpha_{t_{2}}, \ldots, \alpha_{t_{n}}$ at times $t_{1}, t_{2}, \ldots, t_{n}$, the joint probability of finding all the associated properties is

$$
\begin{aligned}
& \operatorname{Prob}\left(\alpha_{t_{1}}=1 \text { at } t_{1} \text { and } \alpha_{t_{2}}=1 \text { at } t_{2} \text { and } \ldots \alpha_{t_{n}}=1 \text { at } t_{n} ; \rho\left(t_{0}\right)\right)= \\
& \quad \operatorname{tr}\left(\alpha_{t_{n}}\left(t_{n}\right) \ldots \alpha_{t_{1}}\left(t_{1}\right) \rho\left(t_{0}\right) \alpha_{t_{1}}\left(t_{1}\right) \ldots \alpha_{t_{n}}\left(t_{n}\right)\right)
\end{aligned}
$$

where we have used the relation $P^{2}=P$ for a projection operator.

The main assumption of the consistent histories interpretation of quantum theory is that, under appropriate conditions, the probability assignment (2.7) is still meaningful for a closed system, with no external observers or associated measurement-induced state-vector reductions (thus signalling a move from 'observables' to 'beables'). The satisfaction or otherwise of these conditions is determined by the behaviour of the decoherence functional $d_{\rho}(\alpha, \beta)$ which, for the pair of sequences of projection operators $\alpha:=\left(\alpha_{t_{1}}, \alpha_{t_{2}}, \ldots, \alpha_{t_{n}}\right)$ and $\beta:=\left(\beta_{t_{1}}, \beta_{t_{2}}, \ldots, \beta_{t_{n}}\right)$ is defined as

$$
d_{\rho}(\alpha, \beta):=\operatorname{tr}\left(\alpha_{t_{n}}\left(t_{n}\right) \ldots \alpha_{t_{2}}\left(t_{2}\right) \alpha_{t_{1}}\left(t_{1}\right) \rho\left(t_{0}\right) \beta_{t_{1}}\left(t_{1}\right) \beta_{t_{2}}\left(t_{2}\right) \ldots \beta_{t_{n}}\left(t_{n}\right)\right)=\operatorname{tr}\left(C_{\alpha} \rho\left(t_{0}\right) C_{\beta}^{\dagger}\right)
$$

where

$$
\begin{aligned}
C_{\alpha} & :=\alpha_{t_{n}}\left(t_{n}\right) \ldots \alpha_{t_{2}}\left(t_{2}\right) \alpha_{t_{1}}\left(t_{1}\right) \\
& =U\left(t_{0}, t_{n}\right) \alpha_{t_{n}} U\left(t_{n}, t_{n-1}\right) \ldots U\left(t_{3}, t_{2}\right) \alpha_{t_{2}} U\left(t_{2}, t_{1}\right) \alpha_{t_{1}} U\left(t_{1}, t_{0}\right)
\end{aligned}
$$

The conditions required for the probability assignments (2.7) to be consistent are summarised below in the (more general) context of the Gell-Mann and Hartle axioms.

It is useful at this point to gather together a number of definitions that are associated with these ideas.

- A homogeneous history Џ) is any time-ordered sequence $\left(\widehat{\alpha}_{t_{1}}, \widehat{\alpha}_{t_{2}}, \ldots, \widehat{\alpha}_{t_{n}}\right)$ of projection operators.

- A homogeneous history $\beta:=\left(\widehat{\beta}_{t_{1}}, \widehat{\beta}_{t_{2}}, \ldots, \widehat{\beta}_{t_{n}}\right)$ is coarser than another history $\alpha:=\left(\widehat{\alpha}_{t_{1}}, \widehat{\alpha}_{t_{2}}, \ldots, \widehat{\alpha}_{t_{n}}\right)$ if, for every $t_{i}, \widehat{\beta}_{t_{i}}<\widehat{\alpha}_{t_{i}}$ where $<$ denotes the usual ordering operation on the space of projection operators, i.e., $\widehat{P}<\widehat{Q}$ means that the range of $\widehat{P}$ is a subspace of the range of $\widehat{Q}$ (this includes the possibility that $\widehat{P}=\widehat{Q}$ so that, in particular, every homogeneous history is trivially coarser than itself). This relation on the set of homogeneous histories is a partial ordering 8 .

- Two homogeneous histories $\alpha:=\left(\widehat{\alpha}_{t_{1}}, \widehat{\alpha}_{t_{2}}, \ldots, \widehat{\alpha}_{t_{n}}\right)$ and $\beta:=\left(\widehat{\beta}_{t_{1}}, \widehat{\beta}_{t_{2}}, \ldots, \widehat{\beta}_{t_{n}}\right)$ are disjoint if, for at least one time point $t_{i}, \widehat{\beta}_{t_{i}}$ is disjoint from $\widehat{\alpha}_{t_{i}}$, i.e., the ranges of these two projection operators are orthogonal subspaces of $\mathcal{H}$.

\footnotetext{
${ }^{7}$ The justification of the term 'homogeneous' will be given in section 3.2.

${ }^{8} \mathrm{~A}$ relation $<$ on a set $X$ is a partial ordering if it satisfies the conditions (i) for all $x \in X, x<x$; (ii) $x<y$ and $y<x$ implies $x=y$; and (iii) $x<y$ and $y<z$ implies $x<z$.
} 
- The unit history assigns the unit operator to any time $t$; the null history assigns the zero operator.

- In calculating a decoherence functional it may be necessary to go outside the class of homogeneous histories to include inhomogeneous histories. A history of this type arises as a logical 'or' (denoted $\vee$ ) operation on a pair of disjoint homogeneous histories $\alpha:=\left(\widehat{\alpha}_{t_{1}}, \widehat{\alpha}_{t_{2}}, \ldots, \widehat{\alpha}_{t_{n}}\right)$ and $\beta:=\left(\widehat{\beta}_{t_{1}}, \widehat{\beta}_{t_{2}}, \ldots, \widehat{\beta}_{t_{n}}\right)$. Such a history $\alpha \vee \beta$ is generally not itself a collection of projection operators (i.e., it is not homogeneous) but, when computing the decoherence functional, it is represented by the operator $\widehat{C}_{\alpha \vee \beta}:=\widehat{C}_{\alpha}+\widehat{C}_{\beta}$. The coarse-graining relations $\alpha<\alpha \vee \beta$ and $\beta<\alpha \vee \beta$ are deemed to apply to this disjoint 'or' operation. The 'negation' operation $\neg$ also usually turns a homogeneous history into an inhomogeneous history, with $\widehat{C}_{\neg \alpha}:=\widehat{1}-\widehat{C}_{\alpha}$.

Note that the collection $\left(\widehat{\alpha}_{t_{1}}, \widehat{\alpha}_{t_{2}}, \ldots, \widehat{\alpha}_{t_{n}}\right)$ of projection operators can itself be viewed as a projection operator on the direct sum $\oplus_{t \in\left\{t_{1}, \ldots, t_{n}\right\}} \mathcal{H}_{t}$ of $n$ copies of the Hilbert space $\mathcal{H}$, with the copies being labelled by the time values $\left\{t_{1}, t_{2}, \ldots, t_{n}\right\}$ at which $\alpha$ is defined. This idea will be generalised in section 3 with a construction in which every history, inhomogeneous as well as homogeneous, is represented by a projection operator on a certain Hilbert space (albeit, not the direct sum $\oplus_{t \in\left\{t_{1}, \ldots, t_{n}\right\}} \mathcal{H}_{t}$ ).

\subsection{The Gell-Mann and Hartle axioms}

The Gell-Mann and Hartle axioms [16] generalise the discussion above by postulating a new approach to quantum theory in which the notion of 'history' is ascribed a fundamental role; i.e., a history may be an irreducible entity in its own right that is not necessarily to be construed as a time-ordered string of single-time propositions. For our purposes, these axioms and definitions are essentially as follows:

1. The fundamental ingredients in the theory are a space of histories and a space of decoherence functionals which are complex-valued functions of pairs of histories.

2. The set of histories possesses a partial order $<$. If $\alpha<\beta$ then $\beta$ is said to be coarser than $\alpha$, or a coarse-graining of $\alpha$; dually, $\alpha$ is a finer than $\beta$, or a fine-graining of $\beta$. Heuristically this means that $\alpha$ provides a more precise specification than $\beta$.

3. There is a notion of two histories $\alpha, \beta$ being disjoint, written $\alpha \perp \beta$. Heuristically, if $\alpha \perp \beta$ then if either $\alpha$ or $\beta$ is 'realised', the other is automatically excluded.

4. There is a unit history 1 (heuristically, the history that is always realised) and a null history 0 (heuristically, the history that is never realised). For all histories $\alpha$ we have $0<\alpha<1$.

\footnotetext{
${ }^{9}$ The exception is when $\alpha$ and $\beta$ differ only in their values $\alpha_{t_{i}}$ and $\beta_{t_{i}}$ at a single time point $t_{i}$. In this case, if the single-time projection operators $\alpha_{t_{i}}$ and $\beta_{t_{i}}$ are disjoint then $\alpha \vee \beta$ is equal to $\alpha$ at all time points except $t_{i}$, where it is $\alpha_{t_{i}}+\beta_{t_{i}}$. In particular, $\alpha \vee \beta$ is still homogeneous.
} 
5. Two histories $\alpha, \beta$ that are disjoint can be combined to form a new history $\alpha \vee \beta$ (heuristically, the history ' $\alpha$ or $\beta$ ').

6. A set of histories $\alpha^{1}, \alpha^{2}, \ldots, \alpha^{N}$ is said to be exclusive if $\alpha^{i} \perp \alpha^{j}$ for all $i, j=$ $1,2, \ldots, N$. The set is exhaustive (or complete) if it is exclusive and if $\alpha^{1} \vee \alpha^{2} \vee$ $\ldots \vee \alpha^{N}=1$.

7. Any decoherence functional $d$ satisfies the following conditions:

(a) $d(0, \alpha)=0$ for all $\alpha$.

(b) Hermiticity: $d(\alpha, \beta)=d(\beta, \alpha)^{*}$ for all $\alpha, \beta$.

(c) Positivity: $d(\alpha, \alpha) \geq 0$ for all $\alpha$.

(d) Additivity: if $\alpha \perp \beta$ then, for all $\gamma, d(\alpha \vee \beta, \gamma)=d(\alpha, \gamma)+d(\beta, \gamma)$.

(e) Normalisation: If $\alpha^{1}, \alpha^{2}, \ldots, \alpha^{N}$ and $\beta^{1}, \beta^{2}, \ldots, \alpha^{M}$ are two complete sets of histories then

$$
\sum_{i=1}^{N} \sum_{j=1}^{M} d\left(\alpha^{i}, \beta^{j}\right)=1 .
$$

In standard quantum theory, the definition (2.8) automatically satisfies all these conditions although the discussions in the literature are sometimes a little vague about the extent to which the conditions apply to histories that are inhomogeneous in addition to those that are homogeneous.

It is important to note that this axiomatic scheme is given a physical interpretation only in relation to consistent sets of histories. A complete set $\mathcal{C}$ of histories is said to be (strongly) consistent with respect to a particular decoherence functional $d$ if $d(\alpha, \beta)=0$ for all $\alpha, \beta \in \mathcal{C}$ such that $\alpha \neq \beta$. Under these circumstances, $d(\alpha, \alpha)$ is given the physical interpretation as the probability that the history $\alpha$ will be 'realised' $\square$. The Gell-Mann and Hartle axioms then guarantee that the usual Kolmogoroff probability sum rules will be satisfied.

The only major difference between the axioms above and those actually given by Gell-Mann and Hartle is that the latter (i) extend the above to include infinite sums; and (ii) invoke a notion of 'fine-grained' histories. The former may well be desirable but it first requires defining what is meant by taking a limit of histories; we shall return briefly to this issue later. A history $\alpha$ is defined to be fine-grained if the only histories $\beta$ for which $\beta<\alpha$ are 0 or $\alpha$ itself. In standard quantum theory, histories of this type arise as time-ordered sequences of projection operators whose ranges are all one-dimensional subspaces of the Hilbert space. However, it is not clear whether the existence of such entities should postulated in general $\square$ or, if so, what additional properties they should possess. A particularly advantageous situation is when the space of histories is atomic in

\footnotetext{
${ }^{10}$ Deciding on the precise meaning of 'realised' is part of the general ontological problem that I wish to avoid discussing in this particular paper.

${ }^{11}$ Even in a Hilbert space situation, 'atoms' of this type would not be present if we were concentrating on histories associated with observables whose spectra was purely continuous.
} 
the sense that there exist so many fine-grained, and disjoint, histories that each history can be written as a unique $V$-sum of these atoms. In this case, the additivity property means that the value of a decoherence functional is determined uniquely by its values on pairs of atoms.

\subsection{History filters and temporal supports in standard quantum logic}

We now begin our detailed development of the Gell-Mann and Hartle axioms with the aim of providing a general quantum-logic theory of histories that incorporates whatever quasi-temporal properties the system may possess. In what follows, the construction of such a general scheme will be motivated by the special example of a history version of standard quantum logic. By this is meant a generalisation of the ideas above in which strings of projection operators are replaced by strings of single-time propositions belonging to the lattice $\mathcal{L}$ of some 'standard' quantum logic theory. The hope is that the algebraic structure thus revealed will provide valuable clues to what should be postulated in the general case.

In standard quantum logic the lattice $\mathcal{L}$ of single-time propositions is highly structured. In particular, it is usually required to be complete, $\square$ orthocomplemented $\left.{ }^{3}\right]$, orthomodular ${ }^{\mathbb{W}}$, atomic ${ }^{-15}$ and with the covering property ${ }^{-1}$. A state on the lattice $\mathcal{L}$ is defined to be a real-valued function $\mu$ on $\mathcal{L}$ that is a 'probability measure' i.e.,

$$
\begin{gathered}
0 \leq \mu(\alpha) \leq 1 \text { for all } \alpha \in \mathcal{L}, \\
\mu(0)=0 \text { and } \mu(1)=1
\end{gathered}
$$

and the countable additivity property that if $\alpha^{1}, \alpha^{2}, \ldots$ is a finite or countable sequence of elements in $\mathcal{L}$ that are pairwise disjoint $\square$ then the series $\mu\left(\alpha^{1} \vee \alpha^{2} \vee \ldots\right)$ converges and

$$
\mu\left(\alpha^{1} \vee \alpha^{2} \vee \ldots\right)=\mu\left(\alpha^{1}\right)+\mu\left(\alpha^{2}\right)+\ldots \ldots
$$

Clearly the space $\mathcal{R}$ of all states is a real convex set, i.e., if $\mu$ and $\nu$ are states then so is $r \mu+(1-r) \nu$ for all real $r$ with $0<r<1$.

One of the main aims of this paper is to find candidates for the 'history analogues' of the lattice $\mathcal{L}$ and the state-space $\mathcal{R}$ of the standard Hamiltonian theory. These analogues

\footnotetext{
${ }^{12} \mathrm{~A}$ lattice is complete if the meet and join operations can be applied to arbitrary subsets of elements. It is countably complete if this can be done for countable subsets.

${ }^{13} \mathrm{~A}$ lattice is orthocomplemented if there is a map $\alpha \mapsto \neg \alpha$ with the property that (i) $\neg(\neg \alpha)=\alpha$, (ii) $\alpha<\beta$ implies $\neg \beta<\neg \alpha$, and (iii) $\alpha \wedge \neg \alpha=0$ and $\alpha \vee \neg \alpha=1$.

${ }^{14} \mathrm{~A}$ lattice is orthomodular if $\alpha<\beta$ implies that $\beta=\alpha \vee(\beta \wedge \neg \alpha)$.

${ }^{15}$ An element $\alpha$ of a lattice is an atom if $0<\beta<\alpha$ implies that $\beta=0$ or $\beta=\alpha$. A lattice is atomic if for any non-zero element $\beta$ there exists an atom $\alpha$ with $\alpha<\beta$.

${ }^{16}$ An element $\gamma$ covers $\beta$ if $\beta<\gamma$ and $\beta<\delta<\gamma$ implies that $\delta=\beta$ or $\delta=\gamma$. An atomic lattice has the covering property if for every $\beta$ and every atom $\alpha$ such that $\alpha \wedge \beta=0$ the element $\alpha \vee \beta$ covers $\beta$.

${ }^{17}$ Two propositions $\alpha$ and $\beta$ are disjoint if $\alpha<\neg \beta$.
} 
of $\mathcal{L}$ and $\mathcal{R}$ will be a space $\mathcal{U P}$ of history-propositions and a space $\mathcal{D}$ of decoherence functionals.

We begin with a careful account of some aspects of the quantum-logic version of the standard history theory sketched above. Thus we consider a system with a lattice $\mathcal{L}$ of single-time propositions and start by defining a history filter to be any finite $\$$ collection $\left(\alpha_{t_{1}}, \alpha_{t_{2}}, \ldots, \alpha_{t_{n}}\right)$ of single-time propositions $\alpha_{t_{i}} \in \mathcal{L}$ which is time-ordered in the sense that $t_{1}<t_{2}<\ldots<t_{n}$. Thus, in the special case where $\mathcal{L}$ is identified with the lattice $P(\mathcal{H})$ of projection operators on a Hilbert space $\mathcal{H}$, a history filter is what we earlier called a homogeneous history. However, to distinguish the general situation from the special case, I will continue to refer to a time-ordered string of projection operators as a 'homogeneous history' and reserve the phrase 'history filter' for either the quantum-logic version of standard quantum theory, or for the general quantum-logic theory.

In the case of standard quantum logic, a history filter is a time-labelled version of what Mittelstaedt and Stachow call a sequential conjunction 24, 39, 40] i.e., it corresponds to the proposition ' $\alpha_{t_{1}}$ is true at time $t_{1}$, and then $\alpha_{t_{2}}$ is true at time $t_{2}$, and then ... and then $\alpha_{t_{n}}$ is true at time $t_{n}$ '. The phrase 'history filter' itself is intended to capture the idea that each single-time proposition $\alpha_{t_{i}}$ in the collection $\left(\alpha_{t_{1}}, \alpha_{t_{2}}, \ldots, \alpha_{t_{n}}\right)$ serves to 'filter out' the properties of the system that are realised in the history of the universe.

It is important to be able to manipulate history filters that are associated with different sets of time points. To this end, it is useful to think of a history filter as something that is defined at every time point but which is 'active' only at a finite subset of points. This can be realised mathematically by defining it to be equal to the trivial proposition at all but the active points. More precisely, in standard quantum logic we shall define a history filter $\alpha$ to be an element of the space $\mathcal{F}(\mathcal{T}, \mathcal{L})$ of maps from the space of time points $\mathcal{T}$ (in the present case, the real line $\mathbb{R}$ ) to the lattice $\mathcal{L}$ with the property that each map is (i) equal nowhere to the null single-time proposition, and (ii) equal to the unit single-time proposition for all but a finite set of $t$ values. It will be convenient to append to this space the null history filter which is defined to be the null single-time proposition at all points $t \in \mathcal{T}$. T9

It follows that, in a standard quantum theory realised on a Hilbert space $\mathcal{H}$, a history filter (i.e., a homogeneous history) is represented by an element $\alpha$ of the space of functions $\mathcal{F}\left(\mathcal{T}, P(\mathcal{H})\right.$ ) where $\widehat{\alpha}_{t}$ (the value of the map $\alpha$ at $t \in \mathcal{T}$ ) is equal to the unit operator for all but a finite set of time points $t \in \mathcal{T}$. Objects of this type can be regarded as projection operators on the weak direct sum $\mathcal{F}(\mathcal{T}, \mathcal{H})$ of $\mathcal{H}$-valued functions on $\mathcal{T}$. 20

\footnotetext{
${ }^{18}$ Circumstances might arise where it is necessary to consider infinite collections of propositions. This is awkward within a purely algebraic formalism but one way of handling this problem will appear later in section 3 .

${ }^{19}$ It is also sometimes convenient to allow history filters that are equal to the null single-time proposition at one or more time points but are non-null at other points: these are all regarded as equivalent to the null history.

${ }^{20}$ The qualification 'weak' refers to the fact that the functions $v: \mathcal{T} \rightarrow \mathcal{H}$ in $\mathcal{F}(\mathcal{T}, \mathcal{H})$ are equal to the
} 
The first of my suggested axioms for a general history theory is that it should be based on a space of history filters, but these will no longer just be time-ordered sequences of single-time propositions. In the context of quantum cosmology, a history filter is a possible 'universe' complete with whatever quasi-temporal attributes it may, or may not, possess. For this reason, the set of all history filters in the general theory will be denoted $\mathcal{U}$; in the case of standard quantum logic we will write $\mathcal{U}(\mathcal{L}):=\mathcal{F}(\mathcal{T}, \mathcal{L})$ to indicate the underlying lattice $\mathcal{L}$ of single-time propositions.

Any quasi-temporal properties of the system should be coded in the mathematical structure of $\mathcal{U}$. Thus we must decide how the propositional structure of the set of history filters can be distinguished from that of a set of fixed-time propositions.

The example of standard Hamiltonian quantum theory suggests that the most characteristic property of a history filter is the possibility of dividing it into two sub-histories, one of which 'follows' the other. Conversely, if a history filter $\beta$ follows another one $\alpha$, they can be combined to give a new filter $\alpha \circ \beta$ that, pace Mittelstaedt and Stachow, can be read as 'history $\alpha$ and then history $\beta$ '. More precisely, we say that a history filter $\beta:=\left(\beta_{t_{1}^{\prime}}, \beta_{t_{2}^{\prime}} \ldots \beta_{t_{m}^{\prime}}\right)$ follows $\alpha:=\left(\alpha_{t_{1}}, \alpha_{t_{2}} \ldots \alpha_{t_{n}}\right)$ if $t_{n}<t_{1}^{\prime}$, and then define the combined history $\alpha \circ \beta$ as 21

$$
\alpha \circ \beta:=\left(\alpha_{t_{1}}, \alpha_{t_{2}}, \ldots, \alpha_{t_{n}}, \beta_{t_{1}^{\prime}}, \beta_{t_{2}^{\prime}}, \ldots, \beta_{t_{m}^{\prime}}\right) .
$$

It is clear that this operation satisfies the associative law

$$
\alpha \circ(\beta \circ \gamma)=(\alpha \circ \beta) \circ \gamma
$$

whenever the history filters $\alpha, \beta$ and $\gamma$ are so related that both sides of this equation are well-defined. Thus the space $\mathcal{U}(\mathcal{L})$ of history filters in standard quantum logic becomes a partial semi-group 22 with respect to the combination law $\circ$.

It is clear that the temporal properties of a history filter $\alpha \in \mathcal{U}(\mathcal{L})$ are encoded in the finite set of time points at which it is active; i.e., the points $t \in \mathcal{T}$ such that $\alpha_{t} \neq 1$. This motivates the following definitions:

1. The set of $t \in \mathcal{T}$ for which $\alpha_{t} \neq 1$ is called the temporal support, or just support, of $\alpha \in \mathcal{U}(\mathcal{L})$, and is denoted $\sigma(\alpha)$.

2. The set of all possible temporal supports will be denoted $\mathcal{S}$; in the present case this is just the set of all finite subsets of $\mathcal{T}=\mathbb{R}$.

null vector in $\mathcal{H}$ for all but a finite set of $t$ values. The scalar product between two such vectors $v, w$ is defined by $\left\langle v, w>:=\sum_{t \in \mathcal{T}}<v_{t}, w_{t}>_{\mathcal{H}}\right.$ where $<,>_{\mathcal{H}}$ denotes the scalar product on the Hilbert space $\mathcal{H}$.

${ }^{21}$ A slight variant is to say that $\beta$ follows $\alpha$ if (i) $t_{n}<t_{1}^{\prime}$, or (ii) $t_{n}=t_{1}^{\prime}$, in which case we must have $\alpha_{t_{n}}=\beta_{t_{1}^{\prime}}$. Then define $\alpha \circ \beta$ by (2.14) if $t_{n}<t_{1}^{\prime}$, and by $\alpha \circ \beta:=\left(\alpha_{t_{1}}, \alpha_{t_{2}}, \ldots, \alpha_{t_{n}}, \beta_{t_{2}^{\prime}}, \ldots, \beta_{t_{m}^{\prime}}\right)$ if $t_{n}=t_{1}^{\prime}$.

${ }^{22} \mathrm{~A}$ set $X$ equipped with a combination law $\circ$ is a semi-group if the combination is associative, i.e., $x \circ(y \circ x)=(x \circ y) \circ z$ for all $x, y, z \in X$. It is a partial semi-group if the combination law can be applied only to certain pairs of elements. 
3. The support of the null history is defined to be the empty subset of $\mathbb{R}$.

Whether or not a history filter $\beta$ follows another history filter $\alpha$ is determined solely by their temporal supports. In fact, the space $\mathcal{S}$ of supports of standard Hamiltonian theory can itself be equipped with the structure of a partial semigroup by saying that the support $s_{2}:=\left\{t_{1}^{\prime}, t_{2}^{\prime}, \ldots, t_{m}^{\prime}\right\}$ follows the support $s_{1}:=\left\{t_{1}, t_{2}, \ldots, t_{n}\right\}$ if $t_{n}<t_{1}^{\prime}$, and then defining the composition as

$$
s_{1} \circ s_{2}:=\left\{t_{1}, t_{2}, \ldots, t_{n}, t_{1}^{\prime}, t_{2}^{\prime}, \ldots, t_{m}^{\prime}\right\} .
$$

The relation between the partial semi-group structures on $\mathcal{U}(\mathcal{L})$ and $\mathcal{S}$ is captured by the observation that the support map $\sigma: \mathcal{U}(\mathcal{L}) \rightarrow \mathcal{S}$ is a homomorphism between these two partial semi-groups, i.e.,

$$
\sigma(\alpha \circ \beta)=\sigma(\alpha) \circ \sigma(\beta)
$$

whenever both sides of the equation are defined.

As a matter of convention we shall define the null support to follow, and preceed, every element $s \in \mathcal{S}$ so that $\alpha \circ 0,0 \circ \alpha, \alpha \circ 1$ and $1 \circ \alpha$ are defined for all $\alpha \in \mathcal{U}(\mathcal{L})$ with values $\alpha \circ 0=0=0 \circ \alpha$ and $\alpha \circ 1=\alpha=1 \circ \alpha$ respectively. Thus the unit history 1 serves as a unit for the semi-group structure of $\mathcal{U}(\mathcal{L})$ while the null history 0 is an absorbing element.

\subsection{History filters and temporal supports in the general case}

The point of the discussion above is to emphasise that the essential temporal properties of the space of history filters $\mathcal{U}(\mathcal{L})$ in standard Hamiltonian quantum theory is reflected in its partial semi-group of supports. This raises the possibility of constructing a 'quasitemporal' theory that is more general than the standard theory and in which the quasitemporal stucture is reflected in the structure of the support space; a theory that was totally 'non-temporal' would have a support space equal to a single point.

Thus we postulate that, in the general case, the space $\mathcal{U}$ of history filters (i.e., possible universes) has the structure of a partial semi-group. If $\alpha$ and $\beta$ are such that they can be combined to form $\alpha \circ \beta$ we shall say that $\alpha$ preceeds $\beta$ (written as $\alpha \triangleleft \beta$ ), or $\beta$ follows $\alpha$. Broadly speaking, the idea is that if $\beta$ follows $\alpha$ then there is some causal influence (perhaps, in a rather unusual sense) of events 'localised' in the history $\alpha$ on those localised in $\beta$. This concept of 'following' is captured in a space $\mathcal{S}$ of temporal supports that is associated with $\mathcal{U}$ and which is also a partial semigroup; the two are related by a homomorphism $\sigma: \mathcal{U} \rightarrow \mathcal{S}$ as in (2.17).

Note that a homomorphism is generally a many-to-one map, and therefore information about a history filter $\alpha$ is lost when it is mapped to $\sigma(\alpha) \in \mathcal{S}$. The key idea is that its image in $\mathcal{S}$ just captures the essential information about any quasi-temporal properties it may possess. This raises the prospect of constructing a tower of semi-groups that 
can interpolate between $\mathcal{U}$ and $\mathcal{S}$. Such a tower would be a series of partial semigroups $\mathcal{U}_{1}, \mathcal{U}_{2}, \ldots, \mathcal{U}_{k}$ and homomorphisms $\sigma_{i}: \mathcal{U}_{i} \rightarrow \mathcal{U}_{i+1}$, with $\sigma_{0}: \mathcal{U} \rightarrow \mathcal{U}_{1}$ and $\sigma_{k}: \mathcal{U}_{k} \rightarrow \mathcal{S}$, such that the support homomorphism $\sigma: \mathcal{U} \rightarrow \mathcal{S}$ factorises into the finite chain

$$
\mathcal{U} \stackrel{\sigma_{0}}{\rightarrow} \mathcal{U}_{1} \stackrel{\sigma_{1}}{\rightarrow} \mathcal{U}_{2} \stackrel{\sigma_{2}}{\rightarrow} \ldots \stackrel{\sigma_{k-1}}{\rightarrow} \mathcal{U}_{k} \stackrel{\sigma_{k}}{\rightarrow} \mathcal{S}
$$

each element of which contains less information than the previous one in the chain.

It should be emphasised that the relation of 'following' may not be transitive; i.e., $\alpha \triangleleft \beta$ and $\beta \triangleleft \gamma$ need not imply that $\alpha \triangleleft \gamma$; in particular, we do not suppose that $\triangleleft$ is a partial order on $\mathcal{U}$. The reason is that the set of events 'localised' in $\beta$ that are causally affected by events in $\alpha$ need not include any events that causally affect events in $\gamma$; an explicit example of this phenomenon will appear in the discussion in section 1 of quantum field theory in a non globally-hyperbolic, curved space-time where the support structure is different from that of standard Hamiltonian theory. We should also not rule out the possibility that a history filter may precede itself, or the existence of pairs of histories $\alpha, \beta$ such that $\alpha \triangleleft \beta$ and $\beta \triangleleft \alpha$, leading to a sort of 'closed time-like loop'.

A more exotic example of a potential support structure is given by the partial semigroup of cobordism equivalence classes. More precisely, given three closed $n$-manifolds $\Sigma_{1}, \Sigma_{2}$ and $\Sigma_{3}$, equivalence classes $c_{1}$ and $c_{2}$ of cobordisms 23 from $\Sigma_{1}$ to $\Sigma_{2}$ and $\Sigma_{2}$ to $\Sigma_{3}$ respectively can be combined to give a well-defined 23 equivalence class $c_{1} \circ c_{2}$ of cobordisms from $\Sigma_{1}$ to $\Sigma_{3}$. If desired, a 'time-direction' can be introduced by declaring one of the pair of boundaries $\Sigma_{1}, \Sigma_{2}$ in a cobordism triple $\left(\Sigma_{1}, \Sigma_{2}, \mathcal{M}\right)$ to be the 'initial' $n$ space and the other to be the 'final' $n$ space, with the requirement that the combination $c_{1} \circ c_{2}$ of cobordisms is permitted only if the final $n$-space of $c_{1}$ is diffeomorphic to the initial $n$-space of $c_{2}$; i.e., this is the condition that $c_{2}$ follows $c_{1}$. A temporalsupport structure based on this cobordism operation would be a natural foundation for a certain type of 'quantum topology' theory. This is another example where the relation of 'following' is clearly not transitive. This is shown in Figure 1 where $B$ follows $A$ and $C$ follows $B$ but $C$ does not follow (i.e., is not cobordant with) $A$ (note that the 'final' boundary of $C$ is the empty set.)

It is worth noting in passing that cobordism theory produces a category in which the objects are closed manifolds and the morphisms are equivalence classes of cobordisms. In fact, category theory is the most general language within which to discuss temporal supports. More precisely, the generic model for a space of temporal supports is based on the morphisms in a category that is small (i.e., the collection of objects is a set rather than a class); this is also related to the general theory of groupoids [17, 21]. This idea (in particular, the example of cobordism theory) will be developed in a later paper.

In summary, the discussion above suggests that a general history theory will contain the following ingredients.

\footnotetext{
${ }^{23} \mathrm{~A}$ cobordism from $\Sigma_{1}$ to $\Sigma_{2}$ is an $n+1$-dimensional manifold $\mathcal{M}$ whose boundary is the disjoint union of submanifolds diffeomorphic to $\Sigma_{1}$ and $\Sigma_{2}$.
} 
1. The basic entity in the theory is a partial semi-group $\mathcal{U}$ of history filters (i.e., possible 'universes').

2. There is an associated partial semi-group $\mathcal{S}$ of temporal supports with a unit element $*$ such that $s \circ *=* \circ s=s$ for all $s \in \mathcal{S}$. A theory with no fundamental 24 temporal properties at all has $\mathcal{S}=\{*\}$.

3. There is a null history 0 and a unit history 1 , both of whose support is *. These can be combined with any history filter $\alpha$ to give

$$
\alpha \circ 1=1 \circ \alpha=\alpha
$$

and

$$
\alpha \circ 0=0 \circ \alpha=0 .
$$

4. The function $\sigma: \mathcal{U} \rightarrow \mathcal{S}$ that maps a history filter to its support is a homomorphism of the partial semi-groups. It may be profitable to factorise it as a tower of semigroups as in (2.18).

\subsection{Nuclear supports and nuclear history filters}

In standard quantum theory, any history filter $\alpha:=\left(\alpha_{t_{1}}, \alpha_{t_{2}}, \ldots, \alpha_{t_{n}}\right)$ with support $\left\{t_{1}, t_{2}, \ldots, t_{n}\right\}$ can be written as the composition

$$
\alpha=\alpha_{t_{1}} \circ \alpha_{t_{2}} \circ \ldots \circ \alpha_{t_{n}}
$$

in which the single-time proposition $\alpha_{t_{i}}, i=1,2, \ldots, n$ is regarded as a history filter whose temporal support is the singleton set $\left\{t_{i}\right\}$.

In a general history theory it is important to know when a history filter can be decomposed into the product of other filters in an analogous way. The following general definitions are useful for this purpose.

1. A history filter $\alpha \in \mathcal{U}$ is nuclear if it cannot be written in the form $\alpha=\alpha_{1} \circ \alpha_{2}$ with both constituent history filters $\alpha_{1}, \alpha_{2} \in \mathcal{U}$ being different from the unit history.

2. A support $s \in \mathcal{S}$ is nuclear if it cannot be written in the form $s=s_{1} \circ s_{2}$ with both constituent supports $s_{1}, s_{2} \in \mathcal{S}$ being different from the unit support $*$.

3. A decomposition of $\alpha$ of the form $\alpha=\alpha_{1} \circ \alpha_{2} \circ \ldots \alpha_{N}$ is irreducible if the constituent history filters $\alpha_{i}, i=1 \ldots N$, are all nuclear.

\footnotetext{
${ }^{24}$ Of course, this does not rule out temporal characteristics 'emerging' in some coarse-grained sense. Indeed, any proper theory of quantum gravity may well be of this type.
} 
Note that, in the decomposition (2.21) in standard quantum theory, the constituents $\alpha_{t_{i}}$ are nuclear histories in the sense above, and $\left\{t_{i}\right\}$ is a nuclear support. Thus, in a general history theory, a nuclear support can be viewed as a (potentially far-reaching) analogue of a 'point of time' in standard Hamiltonian quantum theory; in particular, it admits no further temporal-type subdivisions. Similarly, a nuclear history filter is a general analogue of a single-time proposition.

\subsection{The partial ordering and 'and' operations on $\mathcal{U}$}

If the set of all propositions about the histories of a system is to take on a coherent algebraic form, it might be expected to be equipped with a lattice structure that is analogous to the lattice $\mathcal{L}$ of single-time propositions in standard quantum theory, and which interfaces appropriately with the semi-group structure of the space of supports. Let us analyse the separate operations in turn for the special case of the history version of standard quantum logic.

The partial ordering on $\mathcal{U}$. In standard quantum theory, a partial ordering can be defined on the space of history filters by saying that a history filter $\alpha \in \mathcal{U}(\mathcal{L}):=\mathcal{F}(\mathcal{T}, \mathcal{L})$ is finer than a history filter $\beta$ if, for all time points $t \in \mathcal{T}$, we have $\alpha_{t}<\beta_{t}$ where $<$ denotes the usual partial-order operation on the lattice $\mathcal{L}$ of fixed-time propositions. We expect such fine- (and coarse-) graining relations to be universal, and therefore postulate that in any general history theory:

1. the set $\mathcal{U}$ of history filters is a partially ordered set;

2. the null and unit filters satisfy $0<\alpha<1$ for all $\alpha \in \mathcal{U}$;

3. if $\alpha \in \mathcal{U}$ can be decomposed as $\alpha=\alpha_{1} \circ \alpha_{2}$ then $\alpha<\alpha_{1}$ and $\alpha<\alpha_{2}$.

The third requirement is natural if we bear in mind that the heuristic meaning of $\alpha<\beta$ is that the proposition that 'universe $\alpha$ is realised' contains more information than the same statement made about $\beta$; in particular, the statement that ' $\alpha_{1}$ and then $\alpha_{2}$ is realised' contains more information than either of the two statements ' $\alpha_{1}$ is realised', or ' $\alpha_{2}$ is realised', alone.

The 'and' operation on $\mathcal{U}$. In standard quantum theory, a natural 'and' operation on a pair of history filters $\alpha:=\left(\alpha_{t_{1}}, \alpha_{t_{2}}, \ldots, \alpha_{t_{n}}\right)$ and $\beta:=\left(\beta_{t_{1}}, \beta_{t_{2}}, \ldots, \beta_{t_{n}}\right)$ in $\mathcal{U}(\mathcal{L})$ can be defined by

$$
\alpha \wedge \beta:=\left((\alpha \wedge \beta)_{t_{1}},(\alpha \wedge \beta)_{t_{2}}, \ldots,(\alpha \wedge \beta)_{t_{n}}\right)
$$

where $(\alpha \wedge \beta)_{t_{i}}:=\alpha_{t_{i}} \wedge \beta_{t_{i}}$ is the 'and' operation on the lattice $\mathcal{L}$. In the case of a Hilbert space representation of $\mathcal{L}$ as a lattice of projection operators $P(\mathcal{H}), \widehat{\alpha}_{t_{i}} \wedge \widehat{\beta}_{t_{i}}$ is defined to be the operator that projects onto the intersection of the ranges of $\widehat{\alpha}_{t_{i}}$ and $\widehat{\beta}_{t_{i}}$. Heuristically, this says that the system gets through history filter $\alpha$ and history filter $\beta$ if, and only if, at each time point it passes the single-time projector in both filters. For this reason we set $\alpha \wedge \beta=0$ if there is some time point $t_{i}$ such that $\alpha_{t_{i}} \wedge \beta_{t_{i}}=0$. 
This definition can be extended readily to history filters with different temporal supports by defining $\alpha \wedge \beta \in \mathcal{F}(\mathcal{T}, \mathcal{L})$ as

$$
(\alpha \wedge \beta)_{t}:=\alpha_{t} \wedge \beta_{t} \text { for all } t \in \mathcal{T} \simeq \mathbb{R}
$$

for all $\alpha, \beta \in \mathcal{U}(\mathcal{L}):=\mathcal{F}(\mathcal{T}, \mathcal{L})$. Of course, for most $t$-values, a history filter is the unit proposition and, for any single-time proposition $P, 1 \wedge P=P \wedge 1=P$.

It is clear from this definition that if $\beta$ follows $\alpha$ then $\alpha \circ \beta=\alpha \wedge \beta$, and this relation is one we expect to hold in the general case. Thus the following axiom can be added for a general history theory:

1. The set $\mathcal{U}$ of history filters is equipped with a binary, associative operation called 'and' (denoted $\wedge$ ) such that, heuristically, to say that $\alpha \wedge \beta$ is realised means that both $\alpha$ and $\beta$ are realised. This operation is commutative, i.e., $\alpha \wedge \beta=\beta \wedge \alpha$ for all $\alpha, \beta \in \mathcal{U}$.

2. This 'and'-operation is compatible with the partial-ordering $<$ so that $\mathcal{U}$ is a meet semi-lattice; i.e., $\alpha \wedge \beta$ is a greatest lower bound for $\alpha$ and $\beta$.

3. If $\beta$ follows $\alpha$ then $\alpha \circ \beta=\alpha \wedge \beta$.

\subsection{The space of history propositions}

The next stage is to give a meaning to the negation $\neg \alpha$ or disjunction $\alpha \vee \beta$ of history filters. These operations should possess various familiar properties. For example, the negation operation should satisfy

$$
\neg(\neg \alpha)=\alpha
$$

and

$$
\neg 1=0 \text {, and } \neg 0=1
$$

where 0 and 1 are the null and unit history filters.

Let us start with standard Hamiltonian quantum theory. Note first that the definition (2.23) is actually the 'and' part of the weak direct product $\prod_{t \in \mathcal{T}} \mathcal{L}_{t}$ of copies of the lattice $\mathcal{L}$ indexed by the elements of $\mathcal{T}$. This suggests that we might look at the 'not' and 'or' operations in this product lattice, which are defined analogously to (2.23) as

$$
(\neg \alpha)_{t}:=\neg\left(\alpha_{t}\right)
$$

and

$$
(\alpha \vee \beta)_{t}:=\alpha_{t} \vee \beta_{t}
$$

where $\alpha_{t} \vee \beta_{t}$ is the usual 'or' operation in the lattice $\mathcal{L}$. In an operator realisation this is the projection onto the closure of the linear span of the ranges of the projection operators $\widehat{\alpha}_{t}$ and $\widehat{\beta}_{t}$; the negation operation is $\neg \widehat{\alpha}_{t}=\widehat{1}-\widehat{\alpha}_{t}$. 
These operations may seem natural but they are not the correct ones for our purposes. For example, if $(a, b)$ and $(c, d)$ are two history filters with the same support $\left\{t_{1}, t_{2}\right\}$, the definition above sets $(a, b) \vee(c, d)$ equal to the history filter $(a \vee c, b \vee d)$, which (naïvely) is true if $a$ or $c$ is true at time $t_{1}$, and then $b$ or $d$ is true at time $t_{2}$. In particular, this would be true if $a$ is true at time $t_{1}$ and then $d$ is true at time $t_{2}$, But this corresponds to the history filter $(a, d)$, whereas what we want to say is that the statement that ' $(a, b) \vee(c, d)$ is realised' means that either the filter $(a, b)$ is realised, or the filter $(c, d)$ is realised.

Similarly, according to the above, $\neg(a, b)=(\neg a, \neg b)$ which is true if and only if both $a$ and $b$ are false, whereas what we are looking for should be true if either $a$ is false or $b$ is false.

The implication is that the sought-for 'or' and 'not' operations do not map history filters into history filters but rather take their values in a larger space of all history propositions, denoted $\mathcal{U P}(\mathcal{L})$. Of course, this conclusion is not new: for example, it is reflected in the fact that, in the standard representation of $\alpha$ by $\widehat{C}_{\alpha}$, the history ' $\alpha$ or $\beta$ ' for disjoint homogeneous histories $\alpha$ and $\beta$ is represented by $\widehat{C}_{\alpha}+\widehat{C}_{\beta}$, which is generally not a product of projection operators and hence is not a representation of a homogeneous history; i.e., there is no homogeneous history $\gamma$ such that $\widehat{C}_{\alpha}+\widehat{C}_{\beta}=\widehat{C}_{\gamma}$. Similarly, $\neg \alpha$ is represented by $\widehat{1}-\widehat{C}_{\alpha}$, which is usually not equal to $\widehat{C}_{\beta}$ for any homogeneous history $\beta$.

In summary, the axioms presented so far for the space $\mathcal{U}$ and its support space $\mathcal{S}$ suggest that, in the general case, $\mathcal{U}$ should be regarded as a subset of a larger space $\mathcal{U P}$ of all history propositions which has the structure of an orthocomplemented lattice. Notions such as disjointness can then be defined in the usual way. For example, $\alpha$ is disjoint from $\beta$ is defined to mean $\alpha<\neg \beta$. We would like to know what other structure $\mathcal{U P}$ should have, in particular what type of orthocomplemented lattice it is (for example: orthomodular, atomic, with the covering property, etc). However, it would be much easier to speculate on such matters if we had a mathematical model for the $\mathcal{U P}$-space of standard quantum theory with the property that all the propositions are represented by projection operators. The next step therefore is to find such a model.

\section{An 'HPO' Version of Standard Quantum Theory}

\subsection{A restatement of the main problem}

In standard quantum logic, a history filter $\alpha$ is a set $\left(\alpha_{t_{1}}, \alpha_{t_{2}}, \ldots, \alpha_{t_{n}}\right)$ of single-time propositions. In a Hilbert space realisation, it is a collection $\left(\widehat{\alpha}_{t_{1}}, \widehat{\alpha}_{t_{2}}, \ldots, \widehat{\alpha}_{t_{n}}\right)$ of projection operators that can be regarded as an element of the direct sum $\oplus_{t \in\left\{t_{1}, \ldots, t_{n}\right\}} B(\mathcal{H})_{t}$ of $n$ copies of the algebra $B(\mathcal{H})$ of bounded operators on the Hilbert space $\mathcal{H}$. As such, it is itself a projection operator, as is to be desired for a representative of a proposition (the proposition is that the history $\alpha$ of the universe is 'realised'). Furthermore, the 'and' 
and partial-ordering operations defined in this product lattice have the desired physical interpretation.

However, as emphasised above, this is not the case for the 'or' and 'not' operations, and therefore the theory must be extended from $\mathcal{U}$ to a bigger space $\mathcal{U P}$ of history propositions. In the standard approach to history quantum theory, this problem is solved by noting that, in the computation of the decoherence functional, only the product operator $\widehat{C}_{\alpha}:=\widehat{\alpha}_{t_{n}}\left(t_{n}\right) \widehat{\alpha}_{t_{n-1}}\left(t_{n-1}\right) \ldots \widehat{\alpha}_{t_{1}}\left(t_{1}\right)$ is involved and, furthermore, the putative join $\alpha \vee \beta$ of two disjoint homogeneous histories can be represented in this sense as the sum $\widehat{C}_{\alpha}+\widehat{C}_{\beta}$. From this perspective, it might seem tempting to work always with the $\widehat{C}_{\alpha}$ operator representative of any homogeneous history $\alpha$ and to perform manipulations on these objects alone. For example, if $\beta$ follows $\alpha$ the representative $C$-operator for $\alpha \circ \beta$ is $\widehat{C}_{\alpha \circ \beta}=\widehat{C}_{\beta} \widehat{C}_{\alpha}$, and if $\alpha$ and $\beta$ are disjoint the representative operator is $\widehat{C}_{\alpha \vee \beta}:=$ $\widehat{C}_{\alpha}+\widehat{C}_{\beta}$. However, for our purposes, such a procedure has several defects. In particular:

- the map $\alpha:=\left(\alpha_{t_{1}}, \alpha_{t_{2}}, \ldots, \alpha_{t_{n}}\right) \mapsto \widehat{C}_{\alpha}$ is many-to-one and therefore much information about $\alpha$ is lost in the process;

- $\widehat{C}_{\alpha}$ is not a projection operator, and therefore it is not part of the propositional lattice associated with the Hilbert space $\mathcal{H}$ on which it is defined. This makes it difficult to know what the $C$-representative is of, for example, $\alpha \vee \beta$ when $\alpha$ and $\beta$ are not disjoint.

The intention now is to construct a different operator representation of standard quantum theory in which every history proposition $i s$ represented by a genuine projection operator, thus enabling the whole of $\mathcal{U} \mathcal{P}$ to be identified with the projection lattice of some new Hilbert space. For reasons that will become clear later, we are obliged at this point to restrict our attention to the special case where $\mathcal{L}=P(\mathcal{H})$, i.e., we must work with a normal Hilbert-space based quantum system rather than a general lattice $\mathcal{L}$. However, the result still strongly reinforces the supposition that, for a general history theory, the space $\mathcal{U P}$ is an ortho-complemented lattice that might be representable by projection operators. I shall call any such mathematical structure an HPO theory, where HPO stands for 'history projection operator'.

\subsection{The HPO-theory for standard quantum theory with a fixed temporal support}

If $\widehat{P}$ and $\widehat{Q}$ are projection operators, the product $\widehat{P} \widehat{Q}$ generally fails to be so because, unless $[\widehat{P}, \widehat{Q}]=0$, it is neither hermitian nor idempotent. This is why $\widehat{C}_{\alpha}$ is generally not a projection operator.

\footnotetext{
${ }^{25}$ The operator $\widehat{C}_{\alpha}$ depends explicitly on the dynamics of the system because it uses the Heisenbergpicture operators $\widehat{\alpha}_{t_{i}}\left(t_{i}\right)$. A dynamics-independent version is the simple product $\widehat{\alpha}_{t_{n}} \widehat{\alpha}_{t_{n-1}} \ldots \widehat{\alpha}_{t_{1}}$ of the original Schrödinger picture operators $\widehat{\alpha}_{t_{i}}$.
} 
However, consider instead the tensor product $\widehat{P} \otimes \widehat{Q}$. The product of operators $\widehat{A} \otimes \widehat{B}$ and $\widehat{C} \otimes \widehat{D}$ is defined as $(\widehat{A} \otimes \widehat{B})(\widehat{C} \otimes \widehat{D}):=\widehat{A} \widehat{C} \otimes \widehat{B} \widehat{D}$, while the adjoint operation is $(\widehat{A} \otimes \widehat{B})^{\dagger}:=\widehat{A}^{\dagger} \otimes \widehat{B}^{\dagger}$. It follows at once that $\widehat{P} \otimes \widehat{Q}$ is a projection operator, and is hence a candidate to represent the two-time homogeneous history $(\widehat{P}, \widehat{Q})$. More generally, if we consider the set $\mathcal{U}_{\left\{t_{1}, \ldots, t_{n}\right\}}$ of all homogeneous histories with (for the moment) a fixed support $\left\{t_{1}, t_{2}, \ldots, t_{n}\right\}$, let us represent any such $\alpha=\left(\widehat{\alpha}_{t_{1}}, \widehat{\alpha}_{t_{2}}, \ldots, \widehat{\alpha}_{t_{n}}\right)$ with the tensor product

$$
\theta\left(\widehat{\alpha}_{t_{1}}, \widehat{\alpha}_{t_{2}}, \ldots, \widehat{\alpha}_{t_{n}}\right):=\widehat{\alpha}_{t_{1}} \otimes \widehat{\alpha}_{t_{2}} \otimes \ldots \otimes \widehat{\alpha}_{t_{n}}
$$

which acts on the tensor-product space $\otimes_{t \in\left\{t_{1}, \ldots, t_{n}\right\}} \mathcal{H}_{t}$ of $n$ copies of $\mathcal{H}$.

That the tensor product appears in a natural way can be seen from the following observation. The homogeneous history $\alpha:=\left(\widehat{\alpha}_{t_{1}}, \widehat{\alpha}_{t_{2}}, \ldots, \widehat{\alpha}_{t_{n}}\right)$ belongs to the direct sum $\oplus_{t \in\left\{t_{1}, \ldots t_{n}\right\}} B(\mathcal{H})_{t}$ of $n$-copies of the operator algebra $B(\mathcal{H})$. In constructing the decoherence functional, the map

$$
\left(\widehat{\alpha}_{t_{1}}, \widehat{\alpha}_{t_{2}}, \ldots, \widehat{\alpha}_{t_{n}}\right) \mapsto \operatorname{tr}\left(\widehat{\alpha}_{t_{n}}\left(t_{n}\right) \widehat{\alpha}_{t_{n-1}}\left(t_{n-1}\right) \ldots \widehat{\alpha}_{t_{1}}\left(t_{1}\right) \widehat{B}\right)
$$

is multilinear with respect to the vector space structure of $\oplus_{t \in\left\{t_{1}, \ldots t_{n}\right\}} B(\mathcal{H})_{t}$ for any $\widehat{B} \in$ $B(\mathcal{H})$. However, the fundamental property of the tensor product of a finite collection of vector spaces $V_{1}, V_{2}, \ldots, V_{n}$ is that any multilinear map $\mu: V_{1} \times V_{2} \times \ldots \times V_{n} \rightarrow W$ to a vector space $W$ factorises uniquely through the tensor product to give the chain of maps

$$
V_{1} \times V_{2} \times \ldots \times V_{n} \stackrel{\theta}{\rightarrow} V_{1} \otimes V_{2} \otimes \ldots \otimes V_{n} \stackrel{\mu^{\prime}}{\rightarrow} W .
$$

Hence the map from $\alpha=\left(\widehat{\alpha}_{t_{1}}, \widehat{\alpha}_{t_{2}}, \ldots, \widehat{\alpha}_{t_{n}}\right)$ to $\widehat{\alpha}_{t_{1}} \otimes \widehat{\alpha}_{t_{2}} \otimes \ldots \otimes \widehat{\alpha}_{t_{n}}$ arises naturally in the histories approach to standard quantum theory.

The map $\theta$ from $\oplus_{t \in\left\{t_{1}, \ldots, t_{n}\right\}} B(\mathcal{H})_{t} \rightarrow \otimes_{t \in\left\{t_{1}, \ldots, t_{n}\right\}} B(\mathcal{H})_{t}$ is many-to-one since, for example, $(\lambda \widehat{A}) \otimes\left(\lambda^{-1} \widehat{B}\right)=\widehat{A} \otimes \widehat{B}$ for all $\lambda \in \mathbb{C}, \lambda \neq 0$. However, when restricted to the subspace $\prod_{t \in\left\{t_{1}, \ldots, t_{n}\right\}} P(\mathcal{H})_{t} \subset \oplus_{t \in\left\{t_{1}, \ldots, t_{n}\right\}} B(\mathcal{H})_{t}$ of projection operators, the map is one-to-one, essentially because if $\widehat{P}$ is a projection operator, $\lambda \widehat{P}(\lambda \neq 0)$ is such only for $\lambda=1$. Thus the first observation is that:

- unlike the standard representation with $\widehat{C}_{\alpha}$, no information about the homogeneous history $\left(\widehat{\alpha}_{t_{1}}, \widehat{\alpha}_{t_{2}}, \ldots, \widehat{\alpha}_{t_{n}}\right)$ is lost by representing it with the tensor product $\widehat{\alpha}_{t_{1}} \otimes$ $\widehat{\alpha}_{t_{2}} \otimes \ldots \otimes \widehat{\alpha}_{t_{n}}$

- unlike $\widehat{C}_{\alpha}$, the operator $\widehat{\alpha}_{t_{1}} \otimes \widehat{\alpha}_{t_{2}} \otimes \ldots \otimes \widehat{\alpha}_{t_{n}}$ is hermitian and idempotent, i.e., it is a projection operator.

The key observation is now the following. If $\widehat{P}$ and $\widehat{Q}$ are projection operators on $\mathcal{H}$ then $\widehat{P} \otimes \widehat{Q}$ is a projection operator on $\mathcal{H} \otimes \mathcal{H}$. Furthermore, if $\widehat{P}$ and $\widehat{Q}$ are disjoint (i.e., if $\widehat{P} \widehat{Q}=\widehat{Q} \widehat{P}=0$ ) then $\widehat{P}+\widehat{Q}$ is a projection operator on $\mathcal{H}$. Now suppose that $\widehat{P}$ and $\widehat{Q}$ are disjoint projection operators on $\mathcal{H}$, as are the pair $\widehat{R}$ and $\widehat{S}$; hence $\widehat{P} \widehat{Q}=\widehat{Q} \widehat{P}=0=\widehat{R} \widehat{S}=\widehat{S} \widehat{R}$. Then $(\widehat{P} \otimes \widehat{R})(\widehat{Q} \otimes \widehat{S})=\widehat{P} \widehat{Q} \otimes \widehat{R} \widehat{S}=0=(\widehat{Q} \otimes \widehat{S})(\widehat{P} \otimes \widehat{R})$ and hence $\widehat{P} \otimes \widehat{R}$ and $\widehat{Q} \otimes \widehat{S}$ are disjoint projection operators on $\mathcal{H} \otimes \mathcal{H}$ (actually, it is 
sufficient that just one of the pairs $(\widehat{P}, \widehat{Q})$ and $(\widehat{R}, \widehat{S})$ is disjoint). It follows that their sum $\widehat{P} \otimes \widehat{R}+\widehat{Q} \otimes \widehat{S}$ is also a projection operator on $\mathcal{H} \otimes \mathcal{H}$. In fact, using the lattice structure on $P(\mathcal{H} \otimes \mathcal{H})$ we get

$$
\widehat{P} \otimes \widehat{R} \vee \widehat{Q} \otimes \widehat{S}=\widehat{P} \otimes \widehat{R}+\widehat{Q} \otimes \widehat{S}
$$

which looks remarkably like the $\widehat{C}_{\alpha}+\widehat{C}_{\beta}$ construction we are trying to emulate.

What this example illustrates is that not all projection operators on $\mathcal{H} \otimes \mathcal{H}$ are simply tensor products of projection operators on $\mathcal{H}$. Those that are, are normally called homogeneous; the remainder are inhomogeneous and include certain sums of homogeneous operators.

The general strategy is now clear. With the aid of the map $\theta$, the operator representation $\prod_{t \in\left\{t_{1}, \ldots t_{n}\right\}} P(\mathcal{H})_{t}$ of the space of homogeneous histories $\mathcal{U}_{\left\{t_{1}, \ldots, t_{n}\right\}}$ with temporal support $\left\{t_{1}, t_{2}, \ldots, t_{n}\right\}$ is embedded in the space $P\left(\otimes_{t \in\left\{t_{1}, \ldots, t_{n}\right\}} \mathcal{H}_{t}\right)$ of projection operators on the Hilbert space $\otimes_{t \in\left\{t_{1}, \ldots, t_{n}\right\}} \mathcal{H}_{t}$. The space $P\left(\otimes_{t \in\left\{t_{1}, \ldots, t_{n}\right\}} \mathcal{H}_{t}\right)$ carries the usual lattice structure of projection operators and is therefore a natural model for the space $\mathcal{U} \mathcal{P}_{\left\{t_{1}, \ldots, t_{n}\right\}}$ of history propositions based on homogeneous histories with support $\left\{t_{1}, t_{2}, \ldots, t_{n}\right\}$. In this model, history filters/homogeneous histories are represented by homogeneous projectors, and a general history proposition is represented by an inhomogeneous projector. This explains why the collection $\left(\widehat{\alpha}_{t_{1}}, \widehat{\alpha}_{t_{2}}, \ldots, \widehat{\alpha}_{t_{n}}\right)$ was refered to earlier as a 'homogeneous' history.

In this approach, decoherence functionals will be computed with the aid of the map $D: \otimes_{t \in\left\{t_{1}, \ldots, t_{n}\right\}} B(\mathcal{H}) \rightarrow B(\mathcal{H})$ defined by

$$
D\left(\widehat{A}_{1} \otimes \widehat{A}_{2} \otimes \ldots \widehat{A}_{n}\right):=\widehat{A}_{n}\left(t_{n}\right) \widehat{A}_{n-1}\left(t_{n-1}\right) \ldots \widehat{A}_{1}\left(t_{1}\right)
$$

on homogeneous operators and then extended by linearity. Thus, on a homogeneous history $\alpha \in \mathcal{U}_{\left\{t_{1}, \ldots, t_{n}\right\}}$, the $C$-map is defined by

$$
\widehat{C}_{\alpha}:=D(\theta(\alpha))
$$

and then extended by linearity to the set of inhomogeneous histories with support $\left\{t_{1}, t_{2}, \ldots, t_{n}\right\}$.

\subsection{The missing logical connectives}

The natural lattice structure on $P\left(\otimes_{t \in\left\{t_{1}, \ldots, t_{n}\right\}} \mathcal{H}_{t}\right)$ can be used to define the missing negation and 'or' operations on $\mathcal{U P}$. For example, in a general lattice of projection operators, the projection operator corresponding to the proposition $\neg \widehat{P}$ is $\widehat{1}-\widehat{P}$. Hence, if $\widehat{R}$ and $\widehat{S}$ are projection operators on the Hilbert space $\mathcal{H}$, the negation of the proposition represented by the projection operator $\widehat{R} \otimes \widehat{S}$ on $\mathcal{H} \otimes H$ is just

$$
\neg(\widehat{R} \otimes \widehat{S})=\widehat{1}-\widehat{R} \otimes \widehat{S}
$$


and so, using the $D$-map defined in $(\widehat{3.5})$, we get $D(\neg(\widehat{R} \otimes \widehat{S}))=\widehat{1}-\widehat{S} \widehat{R}$. Thus, for the homogeneous history $\alpha \in \mathcal{U}_{\left\{t_{1}, \ldots, t_{n}\right\}}$, 3.5 3.6) give

$$
\widehat{C}_{\neg \alpha}=\widehat{1}-\widehat{\alpha}_{t_{n}}\left(t_{n}\right) \widehat{\alpha}_{t_{n-1}}\left(t_{n-1}\right) \ldots \widehat{\alpha}_{t_{1}}\left(t_{1}\right)
$$

in accord with the usual identification made in work on decohering histories.

If $\alpha$ and $\beta$ are disjoint homogeneous histories with the same support $\left\{t_{1}, t_{2}, \ldots, t_{n}\right\}$ then, according to the philosophy above, the history proposition $\alpha \vee \beta$ is represented by the sum $\theta(\alpha)+\theta(\beta)=\widehat{\alpha}_{t_{1}} \otimes \widehat{\alpha}_{t_{2}} \otimes \ldots \otimes \widehat{\alpha}_{t_{n}}+\widehat{\beta}_{t_{1}} \otimes \widehat{\beta}_{t_{2}} \otimes \ldots \otimes \widehat{\beta}_{t_{n}}$, and so

$$
\widehat{C}_{\alpha \vee \beta}:=D(\theta(\alpha) \vee \theta(\beta))=D\left(\widehat{\alpha}_{t_{1}} \otimes \widehat{\alpha}_{t_{2}} \otimes \ldots \otimes \widehat{\alpha}_{t_{n}}+\widehat{\beta}_{t_{1}} \otimes \widehat{\beta}_{t_{2}} \otimes \ldots \otimes \widehat{\beta}_{t_{n}}\right)=\widehat{C}_{\alpha}+\widehat{C}_{\beta}
$$

as required. However, we can now describe the $C$-representation of the proposition $\alpha \vee \beta$ when $\alpha$ and $\beta$ are not disjoint. It is simply the image under the map $D$ : $\otimes_{t \in\left\{t_{1}, \ldots t_{n}\right\}} B(\mathcal{H}) \rightarrow B(\mathcal{H})$ of the projection operator $\theta(a) \vee \theta(b)$, i.e., of the operator on $\otimes_{t \in\left\{t_{1}, \ldots t_{n}\right\}} \mathcal{H}$ that projects onto the smallest closed subspace containing the images of the projection operators $\theta(\alpha)=\widehat{\alpha}_{t_{1}} \otimes \widehat{\alpha}_{t_{2}} \otimes \ldots \otimes \widehat{\alpha}_{t_{n}}$ and $\theta(\beta)=\widehat{\beta}_{t_{1}} \otimes \widehat{\beta}_{t_{2}} \otimes \ldots \otimes \widehat{\beta}_{t_{n}}$.

There are situations other than disjointness in which this operator can be written in a simple form. For example, if $\widehat{P}$ and $\widehat{Q}$ are projection operators that commute, but are not disjoint, then it is a standard result that $\widehat{P} \vee \widehat{Q}=\widehat{P}+\widehat{Q}-\widehat{P} \widehat{Q}$. Now suppose that $\left(\widehat{\alpha}_{t_{1}}, \widehat{\alpha}_{t 2}\right)$ and $\left(\widehat{\beta}_{t_{1}}, \widehat{\beta}_{t_{2}}\right)$ are a pair of homogeneous histories with support $\left\{t_{1}, t_{2}\right\}$ (two time points for simplicity only) and which are such that $\left[\widehat{\alpha}_{t_{1}}, \widehat{\beta}_{t_{1}}\right]=0=\left[\widehat{\alpha}_{t_{2}}, \widehat{\beta}_{t_{2}}\right]$. Then the tensor-product projection operators $\widehat{\alpha}_{t_{1}} \otimes \widehat{\alpha}_{t_{2}}$ and $\widehat{\beta}_{t_{1}} \otimes \widehat{\beta}_{t_{2}}$ also commute, and hence

$$
\begin{array}{r}
\widehat{\alpha}_{t_{1}} \otimes \widehat{\alpha}_{t_{2}} \vee \widehat{\beta}_{t_{1}} \otimes \widehat{\beta}_{t_{2}}=\widehat{\alpha}_{t_{1}} \otimes \widehat{\alpha}_{t_{2}}+\widehat{\beta}_{t_{1}} \otimes \widehat{\beta}_{t_{2}}-\left(\widehat{\alpha}_{t_{1}} \otimes \widehat{\alpha}_{t_{2}}\right)\left(\widehat{\beta}_{t_{1}} \otimes \widehat{\beta}_{t_{2}}\right) \\
=\widehat{\alpha}_{t_{1}} \otimes \widehat{\alpha}_{t_{2}}+\widehat{\beta}_{t_{1}} \otimes \widehat{\beta}_{t_{2}}-\widehat{\alpha}_{t_{1}} \widehat{\beta}_{t_{1}} \otimes \widehat{\alpha}_{t_{2}} \widehat{\beta}_{t_{2}}
\end{array}
$$

so that

$$
\widehat{C}_{\alpha \vee \beta}=\widehat{\alpha}_{t_{2}}\left(t_{2}\right) \widehat{\alpha}_{t_{1}}\left(t_{1}\right)+\widehat{\beta}_{t_{2}}\left(t_{2}\right) \widehat{\beta}_{t_{1}}\left(t_{1}\right)-\widehat{\alpha}_{t_{2}}\left(t_{2}\right) \widehat{\beta}_{t_{2}}\left(t_{2}\right) \widehat{\alpha}_{t_{1}}\left(t_{1}\right) \widehat{\beta}_{t_{1}}\left(t_{1}\right)
$$

which, perhaps, is not such an obvious result.

Note that the tensor product structure is well-adapted to discuss the sequential-logic operations introduced by MittelStaedt [24] and Stachow [39. For example, a simple, time-labelled, sequential disjunction is of the form 'proposition $P$ at time $t_{1}$ or then proposition $Q$ at time $t_{2}$ ', denoted $P \sqcup Q$. In terms of history filters, the proposition ' $P$ at time $t_{1}$ ' says nothing about time $t_{2}$ and is therefore represented by the tensor product operator $\widehat{P} \otimes \widehat{1}$. Similarly, the proposition ' $Q$ at time $t_{2}$ ', says nothing about $t_{1}$, and is therefore represented by the operator $\widehat{1} \otimes \widehat{Q}$. Thus, the sequential disjunction $P \sqcup Q$ can be identified with the operator $\widehat{P} \otimes \widehat{1} \vee \widehat{1} \otimes \widehat{Q}$.

Now, even if $\widehat{P}$ and $\widehat{Q}$ do not commute, we still have $[\widehat{P} \otimes \widehat{1}, \widehat{1} \otimes \widehat{Q}]=0$ and hence

$$
\widehat{P} \otimes \widehat{1} \vee \widehat{1} \otimes \widehat{Q}=\widehat{P} \otimes \widehat{1}+\widehat{1} \otimes \widehat{Q}-(\widehat{P} \otimes \widehat{1})(\widehat{1} \otimes \widehat{Q})=\widehat{P} \otimes \widehat{1}+\widehat{1} \otimes \widehat{Q}-\widehat{P} \otimes \widehat{Q}
$$


so that, under the $C$ map, we get

$$
\widehat{C}_{P \sqcup Q}=\widehat{P}+\widehat{Q}-\widehat{Q} \widehat{P}
$$

in agreement with Stachow's result.

Similarly, the sequential conjunction ' $P$ at time $t_{1}$ and then $Q$ at time $t_{2}$ ' (denoted $P \sqcap Q$ ) is represented by $\widehat{P} \otimes \widehat{1} \wedge \widehat{1} \otimes \widehat{Q}$ which is equal to $\widehat{P} \otimes \widehat{Q}$, i.e., the image under $\theta$ of the history filter $(P, Q)$. Thus, in the case of standard quantum theory, a sequential conjunction (with temporal labels) is indeed the same thing as a history filter/homogeneous history.

Finally, it is now clear why we had to restrict our attention at the beginning of this section to the special case $\mathcal{L}=P(\mathcal{H})$. This is because it is not possible to give a sensible definition of the tensor product of a general lattice $\mathcal{L}$ with itself; a well-known problem in standard quantum logic.

\subsection{The HPO-theory for general supports}

The theory developed above works well but, as it stands, it applies only to homogeneous histories with a fixed temporal support $\left\{t_{1}, t_{2}, \ldots, t_{n}\right\}$ and to those general history propositions that can be derived from them. To incorporate arbitrary supports we need to collect together the operator algebras $\otimes_{t \in s} B(\mathcal{H})_{t}$ for all supports $s \in \mathcal{S}$. The natural way of doing this is to use an infinite tensor product of copies of $B(\mathcal{H})$.

Let $\Omega$ denote a family of unit vectors in the Cartesian product $\prod_{t \in \mathcal{T}} \mathcal{H}_{t}$ of copies of $\mathcal{H}$ labelled by the time values $t \in \mathcal{T}$; i.e., $\Omega$ is a map from $\mathcal{T}$ to the unit sphere in $\mathcal{H}$. Recall that the infinite tensor product $\otimes_{t \in \mathcal{T}}^{\Omega} \mathcal{H}_{t}$ based on $\Omega$ is defined to be the set of functions $v: \mathcal{T} \rightarrow \mathcal{H}$ with the property that $v(t)=\Omega(t)$ for all but a finite number of $t$-values. The set of all such functions is given the usual point-wise vector space structure by defining $(a v+b w)(t):=a v(t)+b w(t), a, b \in \mathbb{C}, v, w \in \otimes_{t \in \mathcal{T}}^{\Omega} \mathcal{H}_{t}$, and the scalar product is

$$
<v, w>:=\prod_{t \in \mathcal{T}}<v(t), w(t)>_{\mathcal{H}}
$$

where $<,>_{\mathcal{H}}$ is the inner product on the Hilbert space $\mathcal{H}$. The right hand side of (3.14) is well defined because only a finite number of terms contribute to the product. It is a standard result that the resulting space is a Hilbert space [12].

An infinite tensor product $\otimes_{t \in \mathcal{T}}^{\Omega} B(\mathcal{H})_{t}$ of operator algebras $B(\mathcal{H})$ is naturally associated with this new vector space. It is defined to be the weak closure (i.e., the closure in the weak operator topology) of the set of all functions from $\mathcal{T}$ to $B(\mathcal{H})$ that are equal to the unit operator for all but a finite set of $t$-values. This definition is geared perfectly to accomodate arbitrary temporal supports, and therefore the set of all projection operators in $\otimes_{t \in \mathcal{T}}^{\Omega} B(\mathcal{H})_{t}$ can be taken as a model for the complete space $\mathcal{U} \mathcal{P}$ of history propositions in a standard Hilbert-space based, quantum theory. Elements of the subspace $\mathcal{U}$ of history filters are identified with the homogeneous projection operators in 
$\otimes_{t \in \mathcal{T}}^{\Omega} B(\mathcal{H})_{t}$, i.e., functions from $\mathcal{T}$ to $P(\mathcal{H})$ that are equal to the unit operator for all but a finite set of $t$-values.

Note that use of the weak closure in forming the complete space $\otimes_{t \in \mathcal{T}}^{\Omega} B(\mathcal{H})_{t}$ provides a precise way of giving meaning to a history with an infinite set of projection operators: it is the weak limit of a convergent sequence of homogeneous histories with finite support.

\subsection{Group actions on the HPO space}

An interesting insight into the possible role of group theory in the general history formalism can be obtained by looking at what happens in the HPO form of standard quantum theory. Thus let $R: \mathcal{T} \rightarrow B(\mathcal{H})$ be any map whose value in $B(\mathcal{H})$ is the unit operator on $\mathcal{H}$ for all but the finite set of time values in some support $s=\left\{t_{1}, t_{2}, \ldots, t_{n}\right\}$. Then

$$
R(s):=R_{t_{1}} \otimes R_{t_{2}} \otimes \ldots \otimes R_{t_{n}}
$$

is a well-defined operator on the tensor product space $\otimes_{t \in \mathcal{T}}^{\Omega} \mathcal{H}_{t}$. Now suppose we are given such a map $s \mapsto R(s)$ for each $s \in \mathcal{S}$. Then it is a trivial consequence of definition (3.15) that if $s^{\prime}:=\left\{t_{1}^{\prime}, t_{2}^{\prime}, \ldots, t_{m}^{\prime}\right\}$ is another support that follows $s$ (i.e., $\left.t_{n}<t_{1}^{\prime}\right)$ then

$$
R(s) R\left(s^{\prime}\right)=R\left(s \circ s^{\prime}\right) .
$$

Thus the map $s \mapsto R(s)$ is a representation of the semi-group $\mathcal{S}$ on the HPO Hilbert space.

Now suppose that each $R_{t_{i}}$ is a unitary operator on $\mathcal{H}$. Then the associated operator $R(s)$ is a unitary operator on $\otimes_{t \in \mathcal{T}}^{\Omega} \mathcal{H}_{t}$, and hence, for any history filter $\alpha$ (with arbitrary temporal support), the map

$$
\widehat{\alpha}_{t_{1}^{\prime}} \otimes \widehat{\alpha}_{t_{2}^{\prime}} \otimes \ldots \otimes \widehat{\alpha}_{t_{m}^{\prime}} \mapsto R(s) \widehat{\alpha}_{t_{1}^{\prime}} \otimes \widehat{\alpha}_{t_{2}^{\prime}} \otimes \ldots \otimes \widehat{\alpha}_{t_{m}^{\prime}} R(s)^{-1}
$$

maps the homogeneous projector $\widehat{\alpha}_{t_{1}^{\prime}} \otimes \widehat{\alpha}_{t_{2}^{\prime}} \otimes \ldots \otimes \widehat{\alpha}_{t_{m}^{\prime}}$ into another such. This extends to a map of inhomogeneous projectors, and in such a way that the lattice structure of the history propositions is preserved. Thus, in the unitary case, the map $s \mapsto R(s)$ provides a representation of the semi-group $\mathcal{S}$ as automorphisms of the lattice of history propositions. This suggests very strongly that, in the general case, an important role will be played by representations of the semi-group of supports $\mathcal{S}$ in the group of automorphisms of the lattice $\mathcal{U P}$.

Note that, in standard quantum theory, a very special example is the choice $R_{t}:=$ $e^{-i t H / \hbar}$. In this case, the tensor product $\widehat{\alpha}_{t_{1}}\left(t_{1}\right) \otimes \widehat{\alpha}_{t_{2}}\left(t_{2}\right) \otimes \ldots \otimes \widehat{\alpha}_{t_{n}}\left(t_{n}\right)$ of Heisenbergpicture projectors can be obtained from the product $\widehat{\alpha}_{t_{1}} \otimes \widehat{\alpha}_{t_{2}} \otimes \ldots \otimes \widehat{\alpha}_{t_{n}}$ of Schrödinger operators by the transform

$$
\widehat{\alpha}_{t_{1}}\left(t_{1}\right) \otimes \widehat{\alpha}_{t_{2}}\left(t_{2}\right) \otimes \ldots \otimes \widehat{\alpha}_{t_{n}}\left(t_{n}\right)=U(s) \widehat{\alpha}_{t_{1}} \otimes \widehat{\alpha}_{t_{2}} \otimes \ldots \otimes \widehat{\alpha}_{t_{n}} U(s)^{-1}
$$

where $s:=\left\{t_{1}, t_{2}, \ldots, t_{n}\right\}$ and $U_{t}:=e^{-i t H / \hbar}$. This suggests that, in the general case, if an HPO representation can be found situations may arise in which a quasi-temporal type of dynamical evolution, together with an associated Heisenberg picture, can be achieved with the aid of a special operator representation of $\mathcal{S}$. 


\subsection{The general axioms for history propositions}

Motivated by the above we can now give a provisional form of the expanded set of history axioms towards which we have been working. These should be viewed as a more detailed version of the original Gell-Mann and Hartle axioms and, hopefully, could be used as a basis for a general analysis of quantum gravity theories in which the standard notions of continuous time and space need not be fundamental ingredients. The general axioms and definitions are as follows.

H1. The space of history filters. The fundamental ingredient in a theory of histories is a space $\mathcal{U}$ of history filters, or possible universes. This space has the following structure.

1. $\mathcal{U}$ is a partially-ordered set with a unit history filter 1 and a null history filter 0 such that $0<\alpha<1$ for all $\alpha \in \mathcal{U}$;

2. $\mathcal{U}$ has a meet operation $\wedge$ which combines with the partial order $<$ to form a meet semi-lattice with unit 1 so that $1 \wedge \alpha=\alpha$ for all $\alpha \in \mathcal{U}$. The null history is absorptive in the sense that $0 \wedge \alpha=0$ for all $\alpha \in \mathcal{U}$.

3. $\mathcal{U}$ is a partial semi-group with composition law denoted o. If $\alpha, \beta \in \mathcal{U}$ can be combined to give $\alpha \circ \beta \in \mathcal{U}$ we say that $\beta$ follows $\alpha$, or $\alpha$ preceeds $\beta$, and write $\alpha \triangleleft \beta$. The $\circ$ and $\wedge$ laws are compatible in the sense that if $\alpha \circ \beta$ is defined then it is equal to $\alpha \wedge \beta$.

4. The null and unit histories can always be combined with any history filter $\alpha$ to give

$$
\begin{aligned}
& \alpha \circ 1=1 \circ \alpha=\alpha \\
& \alpha \circ 0=0 \circ \alpha=0 .
\end{aligned}
$$

H2. The space of temporal supports. Any quasi-temporal properties of the system are encoded in a partial semi-group $\mathcal{S}$ of supports with unit $*$. The support space has the following properties.

1. There is a semi-group homomorphism $\sigma: \mathcal{U} \rightarrow \mathcal{S}$ that assigns a support to each history filter. The support of 0 and 1 is defined to be $* \in \mathcal{S}$.

2. A history filter $\alpha$ is nuclear if it has no non-trivial decomposition of the form $\alpha=\beta \circ \gamma, \beta, \gamma \in \mathcal{U}$; a temporal support $s$ is nuclear if it has no non-trivial decomposition of the form $s=s_{1} \circ s_{2}, s_{1}, s_{2} \in \mathcal{S}$. Nuclear supports are the analogue of points of time; nuclear history filters are the analogue of single-time propositions.

A decomposition of $\alpha \in \mathcal{U}$ as $\alpha=\alpha^{1} \circ \alpha^{2} \circ \ldots \circ \alpha^{N}$ is irreducible if the constituent history filters $\alpha^{i} \in \mathcal{U}, i=1,2, \ldots, N$ are all nuclear. 
3. A resolution of the semi-group homomorphism $\sigma: \mathcal{U} \rightarrow \mathcal{S}$ is a chain of semigroups $\mathcal{U}_{i}$ and semi-group homomorphisms $\sigma_{i}$ so that $\sigma$ factors as the composition

$$
\mathcal{U} \stackrel{\sigma_{0}}{\rightarrow} \mathcal{U}_{1} \stackrel{\sigma_{1}}{\rightarrow} \mathcal{U}_{2} \stackrel{\sigma_{2}}{\rightarrow} \ldots \stackrel{\sigma_{k-1}}{\rightarrow} \mathcal{U}_{k} \stackrel{\sigma_{k}}{\rightarrow} \mathcal{S} .
$$

H3. The space of history propositions. The space $\mathcal{U}$ of history filters is embedded in a larger space $\mathcal{U P}$ of history propositions. This space is a ortho-complemented lattice with a structure that is consistent with the semi-lattice structure on the subspace $\mathcal{U}$. One may also require the lattice to be countably complete, or even complete, depending on its cardinality. In addition:

1. The space $\mathcal{U P}$ can be generated from $\mathcal{U}$ by the application of a finite (or, perhaps, countably infinite) number of $\neg, \vee$ and $\wedge$ lattice operations. This captures the idea that elements of $\mathcal{U P}$ represent propositions 'about' history filters (i.e., about possible universes).

2. An important role may be played by representations of the partial semi-group $\mathcal{S}$ in the automorphism group of the lattice $\mathcal{U P}$.

3. Any representation of the ortho-complemented lattice $\mathcal{U P}$ by projection operators on a Hilbert space is called an HPO quantisation of the system. In such a quantisation, some of the automorphisms mentioned above might be implemented with unitary transformations.

4. Two history propositions $\alpha$ and $\beta$ are said to be disjoint, denoted $\alpha \perp \beta$, if $\alpha<\neg \beta$. A set of history propositions $\left\{\alpha^{1}, \alpha^{2}, \ldots, \alpha^{N}\right\}$ is exclusive if its elements are pairwise disjoint. It is exhaustive (or complete) if $\alpha^{1} \vee \alpha^{2} \vee \ldots \vee \alpha^{N}=1$. Countable sets (i.e., with $N=\infty$ ) are permitted where appropriate.

H4. The space of decoherence functionals. A decoherence functional is a complexvalued map of pairs of history propositions; the set of all such maps is denoted $\mathcal{D}$. Any decoherence functional has the following properties:

1. The 'inner-product' type conditions:

- Hermiticity: $d(\alpha, \beta)=d(\beta, \alpha)^{*}$ for all $\alpha, \beta \in \mathcal{U P}$.

- Positivity: $d(\alpha, \alpha) \geq 0$ for all $\alpha \in \mathcal{U P}$.

- Null triviality: $d(0, \alpha)=0$ for all $\alpha \in \mathcal{U P}$.

2. Conditions related to the potential probabilistic interpretation:

- Additivity: if $\alpha \perp \beta$ are general history propositions then, for all $\gamma \in \mathcal{U P}$, $d(\alpha \vee \beta, \gamma)=d(\alpha, \gamma)+d(\beta, \gamma)$ 
- Normalisation $20: d(1,1)=1$

3. There may be a natural topology on $\mathcal{U P}$ such that each decoherence functional $d \in \mathcal{D}$ is a continuous ${ }^{27}$ function of its arguments.

H5. The physical interpretation of these axioms is the same as that of the Gell-Mann and Hartle axioms, i.e., the diagonal element $d(\alpha, \alpha)$ is interpreted as the probability of the history proposition $\alpha$ being 'true' when $\alpha$ is part of a consistent set. If this is not the case, no direct physical meaning is ascribed to the real number $d(\alpha, \alpha)$.

The axioms above might be tightened or relaxed in a variety of ways; certainly one would not wish to be too pedantic at this stage of the development. For example, Dowker and Halliwell [5] have pointed out that the decoherence functional (2.8) in standard Hamiltonian quantum theory obeys the Schwarz-type inequality

$$
|d(\alpha, \beta)| \leq d(\alpha, \alpha)^{\frac{1}{2}} d(\beta, \beta)^{\frac{1}{2}}
$$

for all history filters $\alpha$ and $\beta$. This might be a good candidate for inclusion as an extra axiom in the case of a general history theory. Note that it renders the 'null triviality' axiom redundant.

In normal quantum theory, the lattice $\mathcal{L}$ of propositions is not only complete and ortho-complemented: it is also orthomodular, atomic and with the covering property. Each of these properties has a direct physical implication, and it is therefore a matter of some interest to explore the extent to which they should also be imposed on the lattice $\mathcal{U P}$ of history theory.

In this context it is appropriate to recall the powerful representation theorems that assert that any complete, orthomodular atomic lattice with the covering property (and of length $\geq 4$ ) can be realised as the lattice of closed subspaces of a vector space $V$ equipped with a hermitian form [36], [2]. If the field over which $V$ is defined is the real, complex or quaternionic numbers then, under a minor continuity requirement on the involution operator (complex conjugation in the complex case), $V$ is a Hilbert space. It follows that if the space $\mathcal{U P}$ of history propositions is a lattice of this type, it can be realised as the lattice $P(\mathcal{V})$ of projection operators on some Hilbert space $\mathcal{V}$, i.e., an 'HPO-theory' necessarily exists. Of course, the HPO-Hilbert space $\mathcal{V}$ need not be anything like the tensor-product structure defined above for standard quantum theory; clearly, this will depend on the properties of the temporal supports. On the other hand, if either the orthomodular or the atomicity condition is absent the possibility arises of finding mathematical models that are not connected with Hilbert spaces at all. This is an intriguing topic for further research.

\footnotetext{
${ }^{26}$ This condition, plus additivity, implies that if $\alpha^{1}, \alpha^{2}, \ldots, \alpha^{N}$ and $\beta^{1}, \beta^{2}, \ldots, \beta^{N}$ are two complete sets of history propositions then $\sum_{i=1}^{N} \sum_{j=1}^{M} d\left(\alpha^{i}, \beta^{j}\right)=1$. In the infinite case one may wish to extend this to allow both $N$ and $M$ to be infinite.

${ }^{27}$ Such a condition holds in standard quantum theory because of the result that $\widehat{A} \mapsto \operatorname{tr}(\widehat{A} \widehat{B})$ is a weakly continuous function on bounded subsets of $B(\mathcal{H})$ for each trace-class operator $\widehat{B}$.
} 


\subsection{The non-applicability of Gleason's theorem to the HPO- theory}

We have seen that all propositions about histories in standard Hamiltonian quantum theory can be represented by projection operators on the Hilbert space $\mathcal{V}$ of the HPOtheory. It is instructive to see how Gleason's theorem [10] looks in this context.

Let $\mu$ be any state on the lattice $P(\mathcal{H})$ of projection operators on a Hilbert space $\mathcal{H}$, i.e., $\mu$ is a real-valued function on $P(\mathcal{H})$ that satisfies the axioms (2.11 2.13). Then Gleason showed that, if $\operatorname{Dim}(\mathcal{H})>3$, there exists a density matrix $\rho_{\mu}$ on $\mathcal{H}$ such that, for all $\alpha \in P(\mathcal{H})$,

$$
\mu(\alpha)=\operatorname{tr}\left(\rho_{\mu} \alpha\right)
$$

which, of course, is the familiar expression for the probability in standard quantum theory.

Gleason's theorem is important because it sharply limits the ways in which the quantum-logical structure contained in the pair $(\mathcal{L}, \mathcal{R})$ can be represented in concrete mathematical terms. More precisely, if the lattice of propositions $\mathcal{L}$ is postulated to be the projection lattice $P(\mathcal{H})$ of some Hilbert space $\mathcal{H}$, then Gleason's theorem shows that the states must necessarily be of the standard form (3.23). Thus, to construct a non-standard type of quantum theory in which states are still additive on all pairs of disjoint propositions it is necessary to start with a model for $\mathcal{L}$ that is not the projection lattice of a Hilbert space.

Consider now the case where $\mathcal{V}$ is the Hilbert space of a general HPO theory, i.e., the set of history propositions $\mathcal{U P}$ is represented by the lattice $P(\mathcal{V})$ of projection operators on $\mathcal{V}$. Each decoherence functional $d \in \mathcal{D}$ defines a real-valued map $\mu_{d}$ on $\mathcal{U P}$ by $\mu_{d}(\alpha):=d(\alpha, \alpha)$. This certainly satisfies the first two requirements (2.11 2.12) of an abstract state and therefore, if this map was additive on all complete sets of history propositions, Gleason's theorem would imply that it is necessarily given by a density matrix acting on $\mathcal{V}$ via (3.23). Under these circumstances, it would be rather difficult to tell the difference between a history theory and a normal (single-time) theory.

However, the central idea behind the notion of consistent histories is precisely that the decoherence functional is not additive in this sense, i.e., even if the histories $\alpha$ and $\beta$ are disjoint, $\mu_{d}(\alpha \vee \beta)=d(\alpha \vee \beta, \alpha \vee \beta)$ may not equal $\mu_{d}(\alpha)+\mu_{d}(\beta)=d(\alpha, \alpha)+d(\beta, \beta)$. In fact, this is generally the case since additivity applies only to the special subsets of 'consistent' histories. Thus Gleason's theorem is not applicable, and so it is feasible to seek decoherence functionals on $P(\mathcal{V})$ that are something other than density matrices on $\mathcal{V}$. Indeed, this feature is exploited already in standard quantum theory since the decoherence functional $d(\alpha, \beta):=\operatorname{tr}\left(\widehat{C}_{\alpha} \widehat{\rho} \widehat{C}_{\beta}^{\dagger}\right)$ cannot be written in terms of a density matrix on the HPO-Hilbert space $\otimes_{t \in \mathcal{T}}^{\Omega} \mathcal{H}_{t}$.

This suggests rather strongly that interesting general history theories might be constructed by postulating the space of history propositions $\mathcal{U P}$ to be the projection lattice $P(\mathcal{V})$ of some Hilbert space $\mathcal{V}$, and then to look for decoherence functionals whose di- 
agonal elements are not density matrices on $\mathcal{V}$. For this reason it is of considerable importance to see what can be said in general about the space of decoherence functionals associated with the projection lattice of a Hilbert space. This is another topic for future research.

\section{Quantum Field Theory in a Curved Space-time}

The application of consistent histories to quantum field theory was discussed by Blencowe [3] in the case of a scalar field $\phi$ propagating on a four-dimensional manifold $\mathcal{M}$ with a globally-hyperbolic metric $\gamma$. Global hyperbolicity guarantees the existence of a global time function that can be used to foliate $\mathcal{M}$ into a one-parameter family, $t \mapsto \Sigma_{t}$, of spacelike hypersurfaces $\Sigma_{t}$ of $\mathcal{M}$. Blencowe defined a history to be a collection of propositions about the values of a sequence $A_{t_{i}}, i=1,2, \ldots, n$ of observables where $A_{t_{i}}$ is localised in a bounded open subset $O_{t_{i}}$ of $\Sigma_{t_{i}}$; these regions can be time-ordered by requiring $t_{1}<t_{2}<\ldots<t_{n}$. Thus the global hyperbolicity of $\gamma$ is used to reduce the problem to something resembling standard Hamiltonian quantum theory.

A more space-time oriented approach was suggested by Hartle [14] who emphasised the importance of observables regarded as averages of field variables over space-time regions. The causal structure involved is related to ideas of Sorkin 38 about statevector reduction in relativistic quantum theory. Other significant work in this area is the general study by Mittelstaedt, and by Mittelstaedt and Stachow [27, 26, 29, 28], of 'relativistic quantum logic': a quantum-logical version of the ideas of space-time localised observables that are much used in $C^{*}$-algebra approaches to quantum field theory.

My aim here is to show briefly how the ideas of Hartle and Sorkin lead naturally to a nice example of a 'temporal support' in a quasi-temporal situation. A lot more could be said about the quantum field theoretical aspects of the theory, but this is deferred to another occasion

We start with a pair $(\mathcal{M}, \gamma)$ where the Lorentzian metric $\gamma$ is not required to be globally hyperbolic (this is one part of the 'quasi-temporal' aspect of the system). The basic propositions in the theory will be of the form that the smeared field $\phi(f)$ lies in some subset $I$ of $\mathbb{R}$ where $f$ is smooth test function on $\mathcal{M}$ (this proposition will be denoted $P(f, I))$. The 'history' concept comes in via the idea of looking at a collection of such propositions in which the supports of the test-functions concerned are localised in space-time regions that are causally related in some way.

To facilitate this idea, let us say that a subset $A \subset \mathcal{M}$ preceeds another subset $B \subset \mathcal{M}$, denoted $A \prec B$, if

$$
\begin{gathered}
A \bigcap B=\emptyset, \\
J^{+}(A) \bigcap B \neq \emptyset
\end{gathered}
$$

and

$$
J^{+}(B) \bigcap A=\emptyset
$$


where the chronological future $J^{+}(A)$ of $A$ is defined as usual to be the set of points in $\mathcal{M}$ that can be reached from $A$ by future-directed, non space-like curves. The purpose of (4.3) is to avoid a situation like that shown in Figure 2 where $A$ and $B$ can each causally affect the other.

As emphasised by Sorkin [38], the relation $\prec$ is not a partial ordering; in particular, $A \prec B$ and $B \prec C$ does not necessarily imply that $A \prec C$. This is because the points in $B$ that causally influence $C$ (i.e., $\left\{b \in B \mid J^{+}(b) \cap C \neq \emptyset\right\}$ ) may not lie in that part of $B\left(B \cap J^{+}(A)\right)$ that intersects the causal future $J^{+}(A)$ of $A$. This is illustrated in Figure 3 .

The basic idea of the history formalism for this theory is that an 'elementary' history filter is a set of propositions $P\left(f_{i}, I_{i}\right), i=1,2, \ldots, n$ in which the support of each $f_{i}$ is compact (so that the proposition is localised in the space-time $\mathcal{M}$ ) and connected. Propositions involving regions that are disconnected can be built up from propositions localised in connected regions. This motivates the following definitions.

1. An open subset $A \subset \mathcal{M}$ is a basic region of $\mathcal{M}$ if $A$ is connected and has a compact closure.

2. A temporal support is a collection $s=\left\{O_{1}, O_{2}, \ldots, O_{n}\right\}$ of basic regions $O_{i}$ whose closures are non-intersecting, and which are such that, for each pair $O_{i}, O_{j} \in s$, either (i) $O_{i} \prec O_{j}$, or (ii) $O_{j} \prec O_{i}$, or (iii) $O_{i}$ and $O_{j}$ are space-like separated.

3. A temporal support $s^{\prime}:=\left\{O_{1}^{\prime}, O_{2}^{\prime}, \ldots, O_{m}^{\prime}\right\}$ is said to follow another such $s:=$ $\left\{O_{1}, O_{2}, \ldots, O_{n}\right\}$, denoted $s \triangleleft s^{\prime}$, if (i) the closures of the basic regions in $s$ and $s^{\prime}$ are pairwise disjoint, and (ii) $\bigcup_{i=1}^{n} O_{i} \prec \bigcup_{j=1}^{m} O_{j}^{\prime}$.

If $s \triangleleft s^{\prime}$, a semi-group combination law is defined as

$$
s \circ s^{\prime}:=\left\{O_{1}, O_{2}, \ldots, O_{n}, O_{1}^{\prime}, O_{2}^{\prime}, \ldots, O_{m}^{\prime}\right\}
$$

4. A history filter is a collection of propositions $P\left(f_{1}, I_{1}\right), P\left(f_{2}, I_{2}\right) \ldots, P\left(f_{n}, I_{n}\right)$ where the support of the test function $f_{i}, i=1, \ldots, n$ is the closure of the element $O_{i}$ of a temporal support $\left\{O_{1}, O_{2}, \ldots, O_{n}\right\}$.

If $\alpha, \beta$ are history filters with $\sigma(\alpha) \triangleleft \sigma(\beta)$, then $\alpha \circ \beta$ is defined as the union of the collections of propositions contained in $\alpha$ and in $\beta$.

Note that if a temporal support $s$ is nuclear, (i.e., if it cannot be written in the form $s_{1} \circ s_{2}$ ) then no $O_{i} \in s$ can proceed or follow any other $O_{j} \in s$. Thus each $O_{i}$ in $s$ must be space-like separated from every other one. Thus the role of a 'time-point' is played by any finite collection of disjoint basic regions that are space-like separated from each other. It is clear that every temporal support $s$ can be written in the form $s=s_{1} \circ s_{2} \circ \ldots \circ s_{N}$ where $s_{i}, i=1 \ldots N$ are nuclear sets. This means that the time space $\mathcal{T}$ (defined to be the collection of all possible nuclear temporal supports) can serve the 
same role here as did the real numbers in our earlier discussion of standard Hamiltonian quantum theory. In particular, an HPO theory can be constructed in which the most general history proposition is represented as a projection operator on the Hilbert space $\otimes_{\tau \in \mathcal{T}}^{\Omega} \mathcal{H}_{\tau}$

The computation of decoherence functionals follows the same line as that discussed by Hartle [14] and by Sorkin [38]. Specifically, in constructing the $C$-representation of a history filter, if test-functions $f_{i}$ and $f_{j}$ have supports associated with basic regions $O_{i}$ and $O_{j}$ with $O_{i} \prec O_{j}$, then the projection operators representing the basic propositions $P\left(f_{i}, I_{i}\right)$ and $P\left(f_{j}, I_{j}\right)$ must appear in the order $\widehat{P}\left(f_{j}, I_{j}\right) \widehat{P}\left(f_{i}, I_{i}\right)$. If $O_{i}$ and $O_{j}$ are spacelike separated then no order for the operators $\widehat{P}\left(f_{i}, I_{i}\right)$ and $\widehat{P}\left(f_{j}, I_{j}\right)$ can be specified, which is consistent only if they commute. So this is the point at which microcausality enters the history version of quantum field theory in a curved space-time.

Specific quantum field theoretic problems will not be addressed here but it should be noted that the approach discussed above does not help with the difficult problem of deciding how concrete theories should actually be constructed. The basic problem is that, viewed canonically, a quantum field theory admits many unitarily inequivalent representations of the canonical commutation relations, and some way is needed to select the specific one that is deemed to be of physical relevance. In the case of quantum field theory in a curved space-time $(\mathcal{M}, \gamma)$, there is no universal way of doing this unless $\gamma$ admits a time-like Killing vector. The analogue in the histories formalism arises when one tries to construct projection operators like $\widehat{P}(f, I)$. Specifically, there will exist infinitely many unitarily inequivalent representations of the lattice structure of $\mathcal{U P}$ formed from a particular HPO-theory.

\section{Conclusions}

We have argued that the collection $\mathcal{U P}$ of all history propositions in a general history theory can be equipped with a lattice structure that is similar in some respects to the lattice of propositions in standard quantum logic. Any quasi-temporal properties of the theory are coded in the space $\mathcal{S}$ of supports associated with the subspace $\mathcal{U}$ of history filters. The support space can be quite exotic, as shown by the cobordism example mentioned briefly in section 2.4. Of course, a Boolean lattice would correspond to a history version of a classical theory, and quantum-mechanical superselection rules would arise in the usual way via the existence of a non-trivial center for the lattice $\mathcal{U P}$.

We have also seen how an 'HPO-theory' exists for standard quantum theory in which every history proposition can be represented as a projection operator on a certain Hilbert space. This provides valuable clues about the possible lattice structure on $\mathcal{U P}$ in the general case and opens the possibility for novel ${ }^{\circ}$ concepts. It also suggests that useful quantum history theories can be obtained using the lattice of projection operators $P(\mathcal{V})$

\footnotetext{
${ }^{28}$ For example, the lattice structure on $\mathcal{U P}$ can be used to provide a notion of two history propositions commuting [2].
} 
on some Hilbert space $\mathcal{V}$ as a model for $\mathcal{U} \mathcal{P}$, with a support space $\mathcal{S}$ attached in some way.

It is clear that much work remains to be done to develop these ideas into a fully effective tool. Some of the major topics are as follows:

The structure of the space $\mathcal{D}$ of decoherence functionals. Very little has been said in this paper about the mathematical structure of the space of decoherence functionals, but this is clearly of great importance. In standard quantum logic, the analogue of $\mathcal{D}$ is the space $\mathcal{R}$ of states/probability measures on the lattice $\mathcal{L}$ of single-time propositions. Much has been written about how the structures of $\mathcal{R}$ and $\mathcal{L}$ intertwine, and a similar analysis should be done for $\mathcal{D}$ and $\mathcal{U P}$.

Another intriguing issue concerns the use of complex numbers. The only number system that plays a basic role in standard quantum logic is the set of real numbers in which states take their values. Indeed, one of the original reasons for interest in the quantum logic programme was a hope that it would clarify the extent to which the complex number field is, or is not, an essential ingredient in quantum theory. The conclusion there was that complex numbers are not essential, but we have forced them on the history formalism by requiring decoherence functionals to be complex valued. The question arises therefore of whether the axioms for decoherence functionals can be changed so that complex numbers do not play such a fundamental role.

A history analogue of the canonical commutation relations. The canonical commutation relations play an important role in standard Hilbert-space based quantum theory. They arise as the Lie algebra of a group of transformations (the Weyl group) of classical phase space and provide the special class of classical observables that can be assigned self-adjoint operator status in such a way that Poisson brackets go into operator commutators. More generally, any classical system with a state space that admits a transitive group of symplectic transformations $G$ can be quantised by looking for irreducible, unitary representations of $G$; the associated self-adjoint representation of the Lie algebra $L(G)$ then provides the preferred class of quantum observables. 29

It is of considerable interest to ask what is the history analogue of a canonical group. In standard quantum theory on a Hilbert space $\mathcal{H}$ a unitary representation of $G$ on $\mathcal{H}$ induces a $G$ action on $P(\mathcal{H})$; irreducibility of the representation is equivalent to the action on $P(\mathcal{H})$ having no fixed points. This idea can be generalised to the space $F(\mathcal{T}, P(\mathcal{H}))$ of history filters by studying the action on this space of the 'gauged' canonical group $F(\mathcal{T}, G)$ ( $c f$ the discussion in section 3.5). In particular, this suggests the idea of a "preferred class' of history filters in which the constituent single-time projection operators all belong to the spectral resolutions of the Lie algebra of $G$. A detailed study of this example could lead to a group-theoretic approach to the actual construction of general history theories. Of course, the notion of a symmetry group will also have a natural

\footnotetext{
${ }^{29}$ This is thinking of a canonical group as a route for quantising a classical system. Another aspect is the uncertainty relations that are associated with the commutators of the generators of $L(G)$. This has been discussed in depth recently by Halliwell and Anderson [1, 13] for the case of the Weyl group.
} 
translation into history terms.

Topological aspects of quantum theory in a history formalism. It is intriguing to wonder how the familiar topological properties of standard quantum theory translate into the history formalism, and whether any of them continue to be of significance in a more general history theory. For example, in the quantum version of a classical system with configuration space $Q$, different flat, complex vector bundles over $Q$ lead to inequivalent quantisations. Such bundles are classified by the group $\operatorname{Hom}\left(\pi_{1}(Q), U(n)\right)$ of $U(n)$ valued homomorphisms of the fundamental group $\pi_{1}(Q)$ of $Q$. How is this structure reflected in the structure of the space of history propositions? Similarly, how does the famous Berry phase appear in a history formalism? These questions may, or may not, be related to the separate issue of whether the spaces $\mathcal{U}$ and $\mathcal{U} \mathcal{P}$ should be given topological structures in their own right and, if so, what role this plays in the general theory.

The use of alternative algebraic structures. The discussion in this paper has been within the context of quantum logic,i.e., the major mathematical tools are to be drawn from the theory of non-distributive lattices. However, in conventional quantum theory other types of algebraic structure have been employed to great effect, and it is important to see if these can be adapted to the general history formalism. A relatively mild example would be to replace the use of projection operators with the more general positive-operatorvalued measures that have been much used in recent discussions of the measurement problem in quantum theory [4]. A more striking challenge would be to find a $C^{*}$-algebra analogue of the general axioms presented in this paper.

\section{Acknowledgements}

I am extremely grateful to Jonathan Halliwell for numerous discussions on the consistent history formalism and for a careful reading of a draft version of this paper. For the latter task I also extend warm thanks to Arley Anderson and to Jim Hartle.

\section{References}

[1] A. Anderson and J.J. Halliwell. An information-theoretic measure of uncertainty due to quantum and thermal fluctuations. 1993. Imperial College preprint IC 92-93/25.

[2] E.G. Beltrametti and G. Cassinelli. The Logic of Quantum Mechanics. AddisonWesley, London, 1981.

[3] M. Blencowe. The consistent histories interpretation of quantum fields in curved spacetime. Ann. Phys. (NY), 211:87-111, 1991.

[4] P. Busch, P.J. Lahti, and P. Mittelstaedt. The Quantum Theory of Measurement. Springer-Verlag, London, 1991. 
[5] H.F. Dowker and J.J. Halliwell. The quantum mechanics of history: The decoherence functional in quantum mechanics. Phys. Rev., D46:1580-1609, 1992.

[6] M. Gell-Mann and J. Hartle. Alternative decohering histories in quantum mechanics. In K.K. Phua and Y. Yamaguchi, editors, Proceedings of the 25th International Conference on High Energy Physics, Singapore, August, 2-8, 1990, Singapore, 1990. World Scientific.

[7] M. Gell-Mann and J. Hartle. Quantum mechanics in the light of quantum cosmology. In S. Kobayashi, H. Ezawa, Y. Murayama, and S. Nomura, editors, Proceedings of the Third International Symposium on the Foundations of Quantum Mechanics in the Light of New Technology, pages 321-343. Physical Society of Japan, Tokyo, 1990.

[8] M. Gell-Mann and J. Hartle. Quantum mechanics in the light of quantum cosmology. In W. Zurek, editor, Complexity, Entropy and the Physics of Information, SFI Studies in the Science of Complexity, Vol. VIII, pages 425-458. Addison-Wesley, Reading, 1990.

[9] M. Gell-Mann and J. Hartle. Classical equations for quantum systems. 1992. UCSB preprint UCSBTH-91-15.

[10] A.M. Gleason. Measures on the closed subspaces of a Hilbert space. Journal of Mathematics and Mechanics, pages 885-893, 1957.

[11] R.B. Griffiths. Consistent histories and the interpretation of quantum mechanics. J. Stat. Phys., 36:219-272, 1984.

[12] A. Guichardet. Symmetric Hilbert Spaces and Related Topics. Springer-Verlag, New York, 1972.

[13] J.J. Halliwell. Quantum-mechanical histories and the uncertainty principle: I. Information-theoretic inequalities. 1993. grqc 9304039.

[14] J. Hartle. The quantum mechanics of cosmology. In S. Coleman, P. Hartle, T. Piran, and S. Weinberg, editors, Quantum Cosmology and Baby Universes. World Scientific, Singapore, 1991.

[15] J. Hartle. Spacetime grainings in nonrelativistic quantum mechanics. Phys. Rev., D44:3173-3195, 1991.

[16] J. Hartle. Spacetime quantum mechanics and the quantum mechanics of spacetime. In Proceedings on the 1992 Les Houches School, Gravitation and Quantisation. 1993.

[17] P.J. Higgins. Categories and Groupoids. Van Nostrand, London, 1971.

[18] C.J. Isham. Canonical quantum gravity and the problem of time. In Proceedings of the NATO Advanced Study Institute, Salamanca, June 1992. Kluwer Academic Publishers, London, 1993. 
[19] J.M. Jauch. Foundations of Quantum Mechanics. Addison-Wesley, London, 1973.

[20] K. Kuchař. Time and interpretations of quantum gravity. In Proceedings of the 4 th Canadian Conference on General Relativity and Relativistic Astrophysics. World Scientific, Singapore, 1992.

[21] K. Mackenzie. Lie Groupoids and Lie Algebroids in Differential Geometry. Cambridge University Press, Cambridge, 1987.

[22] G.W. Mackey. The Mathematical Foundations of Quantum Mechanics. W.A. Benjamin, New York, 1963.

[23] J. Milnor. Lectures on the h-Cobordism Theorem. Princeton University Press, Princeton, 1965.

[24] P. Mittelstaedt. Time dependent propositions and quantum logic. Jour. Phil. Logic, 6:463-472, 1977.

[25] P. Mittelstaedt. Quantum Logic. D. Reidel, Holland, 1978.

[26] P. Mittelstaedt. Analysis of the EPR-experiment by relativistic quantum logic. In Proceedings of the International Symposium on the Foundations of Quantum Mechanics, pages 251-255. 1983.

[27] P. Mittelstaedt. Relativistic quantum logic. Int. J. Theor. Phys., 22:293-314, 1983.

[28] P. Mittelstaedt. EPR-Paradox, quantum logic and relativity. In P. Lahti and P. Mittelstaedt, editors, Symposium on the Foundations of Modern Physics, pages 171-186. World Scientific, Singapore, 1985.

[29] P. Mittelstaedt and E.W. Stachow. Analysis of the EPR-experiment by relativistic quantum logic. Int. J. Theor. Phys., 22:517-540, 1983.

[30] R. Omnès. Logical reformulation of quantum mechanics. I. Foundations. J. Stat. Phys., 53:893-932, 1988.

[31] R. Omnès. Logical reformulation of quantum mechanics. II. Interferences and the Einstein-Podolsky-Rosen experiment. J. Stat. Phys., 53:933-955, 1988.

[32] R. Omnès. Logical reformulation of quantum mechanics. III. Classical limit and irreversibility. J. Stat. Phys., 53:957-975, 1988.

[33] R. Omnès. Logical reformulation of quantum mechanics. III. Projectors in semiclassical physics. J. Stat. Phys., 57:357-382, 1989.

[34] R. Omnès. From Hilbert space to common sense: A synthesis of recent progress in the interpretation of quantum mechanics. Ann. Phys. (NY), 201:354-447, 1990. 
[35] R. Omnès. Consistent interpretations of quantum mechanics. Rev. Mod. Phys., 64:339-382, 1992.

[36] C. Piron. Foundations of Quantum Physics. W.A. Benjamin, London, 1976.

[37] P. Pták and S. Pulmannová. Orthomodular Structures as Quantum Logics. Kluwer Academic Publishers, London, 1991.

[38] R.D. Sorkin. Impossible measurements on quantum fields. In B.S. Hu and T.A. Jacobson, editors, Directions in General Relativity, Vol. II: a Collection of Essays in honour of Dieter Brill's Sixtieth Birthday. Cambridge University Press, Cambridge, 1993.

[39] E.-W. Stachow. Logical foundations of quantum mechanics. Int. J. Theor. Phys., 19:251-304, 1980.

[40] E.-W. Stachow. Sequential quantum logic. In E.G. Beltrametti and B.C. van Fraassen, editors, Current Issues in Quantum Logic, pages 173-191. Plenum Press, New York, 1981.

[41] J. von Neumann and G. Birkhoff. The logic of quantum mechanics. Annals of Mathematics, 37:823-843, 1936. 